\title{
3D-2D analysis of a thin film with periodic microstructure
}

\author{
J.-F. Babadjian and M. Baía
}

\begin{abstract}
The purpose of this article is to study the behavior of a heterogeneous thin film whose microstructure oscillates on a scale that is comparable to that of the thickness of the domain. The argument is based on a $3 \mathrm{D}-2 \mathrm{D}$ dimensional reduction through a $\Gamma$-convergence analysis, techniques of two-scale convergence and a decoupling procedure between the oscillating variable and the in-plane variable.
\end{abstract}

Keywords: dimension reduction, thin films, periodic integrands, $\Gamma$-convergence, two-scale convergence, equi-integrability.

MSC 2000 (AMS): 35E99, 35M10, 49J45, 74B20, 74K35, 74G65

\section{Introduction and main result}

In this work we study the asymptotic behavior of a heterogeneous $\varepsilon$-thin domain with periodic microstructure of period $\varepsilon$, as $\varepsilon$ goes to zero, through a $\Gamma$-limit analysis. Techniques of two-scale convergence and a decoupling procedure between the oscillating variable and the in-plane variable are used to derive the relaxed two-dimensional energy from its three-dimensional counterpart.

Let $\omega$ be an open and bounded subset of $\mathbb{R}^{2}$. For each $0<\varepsilon \ll 1$ define $\Omega_{\varepsilon}:=\omega \times(-\varepsilon, \varepsilon)$. Consider a deformable thin body occupied by a hyperelastic material with a periodic microstructure of period $\varepsilon$ whose reference configuration is given by the thin domain $\Omega_{\varepsilon}$, and whose stored energy density $W(\varepsilon): \Omega_{\varepsilon} \times \mathbb{R}^{3 \times 3} \rightarrow \mathbb{R}$ is assumed to be a Carathéodory function satisfying some $p$-growth and coercivity conditions $(1<p<\infty)$. We assume that the body is clamped on the lateral boundary, that is $u(x)=x$ on $\partial \omega \times(-\varepsilon, \varepsilon)$ for all admissible deformations of the body, and that it is submitted to the action of regular surface traction densities $g(\varepsilon)$ on $\Sigma_{\varepsilon}:=\omega \times\{-\varepsilon, \varepsilon\}$ and regular dead loads $f(\varepsilon)$. The total energy of this body under the action of this forces is nothing but the difference between the elastic energy and the work of external forces. More precisely

$$
\mathcal{E}(\varepsilon)(u):=\int_{\Omega_{\varepsilon}} W(\varepsilon)(x ; D u) d x-\int_{\Omega_{\varepsilon}} f(\varepsilon) \cdot u d x-\int_{\Sigma_{\varepsilon}} g(\varepsilon) \cdot u d \mathcal{H}^{2},
$$

for $u \in \mathcal{V}(\varepsilon):=\left\{u \in W^{1, p}\left(\Omega_{\varepsilon} ; \mathbb{R}^{3}\right): u(x)=x\right.$ on $\left.\partial \omega \times(-\varepsilon, \varepsilon)\right\}$, and where $\mathcal{H}^{2}$ stands for the two-dimensional Hausdorff measure. It may occur that the minimization problem associated with this energy admits no solution over the set of kinematically admissible fields $\mathcal{V}(\varepsilon)$. However, we can introduce the notion of quasi-minimizer of $\mathcal{E}(\varepsilon), u(\varepsilon) \in \mathcal{V}(\varepsilon)$, by requiring that

$$
\mathcal{E}(\varepsilon)(u(\varepsilon)) \leqslant \inf _{u \in \mathcal{V}(\varepsilon)} \mathcal{E}(\varepsilon)(u)+\varepsilon h(\varepsilon),
$$

where $h(\varepsilon) \searrow 0^{+}$when $\varepsilon \rightarrow 0$. Note that if the minimization problem admits a solution - for instance if $W(\varepsilon)$ is quasiconvex in its second variable - then we can take $h \equiv 0$.

As usual, in order to study this problem as $\varepsilon \rightarrow 0$ we rescale the $\varepsilon$-thin body into a reference domain of unit thickness (see e.g. Anzellotti, Baldo and Percivale [3], Le Dret and Raoult [17], Braides, Fonseca and Francfort 
[10]), so that the resulting energy will be defined on a fixed body, while the dependence on $\varepsilon$ turns out to be explicit in the transverse derivative. For this, we consider the change of variables

$$
\Omega_{\varepsilon} \rightarrow \Omega:=\omega \times I, \quad\left(x_{1}, x_{2}, x_{3}\right) \mapsto\left(x_{1}, x_{2}, \frac{1}{\varepsilon} x_{3}\right),
$$

and define $v\left(x_{\alpha}, \frac{x_{3}}{\varepsilon}\right)=u\left(x_{\alpha}, x_{3}\right)$ on the rescaled cylinder $\Omega$, where $I:=(-1,1)$ and $x_{\alpha}:=\left(x_{1}, x_{2}\right)$ is the in-plane variable. It is well known that the membrane theory arises at the order $\varepsilon$ of a formal asymptotic expansion (see Fox, Raoult and Simo [16]), provided that the body forces are of order 1 and the surface loadings are of order $\varepsilon$. Since this energy is of order $\varepsilon$ we divide the total energy by $\varepsilon$ and, in addition we assume that

$$
\left\{\begin{array}{l}
f(\varepsilon)\left(x_{\alpha}, \varepsilon x_{3}\right)=f\left(x_{\alpha}, x_{3}\right) \\
g(\varepsilon)\left(x_{\alpha}, \varepsilon x_{3}\right)=\varepsilon g\left(x_{\alpha}, x_{3}\right)
\end{array}\right.
$$

where $f \in L^{p^{\prime}}\left(\Omega ; \mathbb{R}^{3}\right), g \in L^{p^{\prime}}\left(\Sigma ; \mathbb{R}^{3}\right)\left(1 / p+1 / p^{\prime}=1\right)$ and $\Sigma:=\omega \times\{-1,1\}$. If $W_{\varepsilon}\left(x_{\alpha}, x_{3} ; \cdot\right)=W(\varepsilon)\left(x_{\alpha}, \varepsilon x_{3} ; \cdot\right)$, for fixed $\varepsilon$ minimizing $\mathcal{E}(\varepsilon)$ on $\mathcal{V}(\varepsilon)$ is equivalent to minimizing

$$
\mathcal{E}_{\varepsilon}(v):=\frac{\mathcal{E}(\varepsilon)(u)}{\varepsilon}=\int_{\Omega} W_{\varepsilon}\left(x ;\left.D_{\alpha} v(x)\right|_{\varepsilon} ^{1} D_{3} v(x)\right) d x-\int_{\Omega} f \cdot v d x-\int_{\Sigma} g \cdot v d \mathcal{H}^{2}
$$

on $\mathcal{V}_{\varepsilon}:=\left\{v \in W^{1, p}\left(\Omega ; \mathbb{R}^{3}\right): v(x)=\left(x_{\alpha}, \varepsilon x_{3}\right)\right.$ on $\left.\partial \omega \times I\right\}$. Denote by $D_{i}=\frac{\partial}{\partial x_{i}}$ for $i \in\{1,2,3\}$ and $D_{\alpha}=$ $\left(D_{1}, D_{2}\right)$. In the sequel, we identify $\mathbb{R}^{d \times N}$ with the space of real $d \times N$ matrices. For all $\bar{\xi}=\left(z_{1} \mid z_{2}\right) \in \mathbb{R}^{3 \times 2}$ and $z \in \mathbb{R}^{3},(\bar{\xi} \mid z)$ is the matrix whose first two columns are $z_{1}$ and $z_{2}$ and whose last one is $z$. Denoting a quasi-minimizer of the rescaled energy by $u_{\varepsilon}\left(x_{\alpha}, x_{3}\right):=u(\varepsilon)\left(x_{\alpha}, \varepsilon x_{3}\right)$, we obtain

$$
\mathcal{E}_{\varepsilon}\left(u_{\varepsilon}\right) \leqslant \inf _{v \in \mathcal{V}_{\varepsilon}} \mathcal{E}_{\varepsilon}(v)+h(\varepsilon) .
$$

Our aim is to study the asymptotic behavior of the equilibrium problem (1.1) as $\varepsilon \rightarrow 0$ via a $\Gamma$-convergence method (we refer to Braides and Defranceschi [9], Braides [11] and Dal Maso [13] for a comprehensive treatment and bibliography on $\Gamma$-convergence).

The motivation for studying problem (1.1) comes from the work of Braides, Fonseca and Francfort [10] who have established an abstract dimensional reduction variational convergence result in a general setting for a family of stored energies of the form $W_{\varepsilon}(x ; \xi)$ and derived specific characterizations for particular cases. In Section 3 of [10] a heterogeneous nonlinear membrane model is derived by $\Gamma$-convergence, and heterogeneity in the transverse direction is considered. Precisely, the authors treat the case where the stored energy density is of the form $W\left(x_{3} ; \xi\right)$, generalizing the previous work of Le Dret and Raoult in [17] who treated a homogeneous material, i.e. when $W$ depends only in $\xi$. Later, Babadjian and Francfort [4] considered energies of the form $W(x ; \xi)$ with a general heterogeneity. Furthermore in Section 4 of $[10]$, a 3D-2D analysis coupled with a homogenization in the in-plane direction is studied in the case where $W_{\varepsilon}(x ; \xi)=W\left(x_{3}, x_{\alpha} / \varepsilon ; \xi\right)$. Shu [22] also investigated similar problems, in the framework of martensitic materials, with different length scales for the film thickness and the material microstructure.

Here we propose to establish a dimensional reduction and homogenization result, where both scales are identical, by adding in the stored energy density an explicit dependence on the in-plane variable $x_{\alpha}$. Namely, we assume that $W_{\varepsilon}\left(x_{\alpha}, x_{3} ; \cdot\right)=W\left(x_{\alpha}, x_{3}, x_{\alpha} / \varepsilon ; \cdot\right)$ for some function $W: \Omega \times \mathbb{R}^{2} \times \mathbb{R}^{3 \times 3} \rightarrow \mathbb{R}$ whose hypotheses will be introduced later.

Two features differentiate our approach from what is available in most of the literature in the subject. The first one is the use of a two-scale convergence argument (see Nguetseng [20, 21] and Allaire [2] for the notion and properties of two-scale convergence). The same argument was used by Baía and Fonseca in [5] in a pure homogenization context, i.e. without considering the dimensional reduction problem. The second feature is connected with the definition of the homogenized stored energy in which two independent variables are occurring : Firstly the integration variable $y_{\alpha}$ and secondly $x_{\alpha}$ on which this function is explicitly depending. 
To take into account this structure, we are led to decouple the oscillating variable from the in-plane variable. This procedure is possible thanks to a continuous extension of Carathéodory functions result introduced by Babadjian and Francfort in [4].

For a comprehensive treatment on the homogenization of integral functionals via a $\Gamma$-limit approach, we refer to Braides and Defransceschi [9] and references therein. We will denote by $\mathcal{L}^{N}$ the $N$-dimensional Lebesgue measure in $\mathbb{R}^{N}$ (in the sequel $N$ will be equal to 2 or 3 ).

For each $\varepsilon>0$ we define $\mathcal{I}_{\varepsilon}: L^{p}\left(\Omega ; \mathbb{R}^{3}\right) \rightarrow \overline{\mathbb{R}}$ by

$$
\mathcal{I}_{\varepsilon}(u):= \begin{cases}\int_{\Omega} W\left(x_{\alpha}, x_{3}, \frac{x_{\alpha}}{\varepsilon} ; D_{\alpha} u(x) \mid \frac{1}{\varepsilon} D_{3} u(x)\right) d x_{\alpha} d x_{3} & \text { if } u \in W^{1, p}\left(\Omega ; \mathbb{R}^{3}\right), \\ +\infty & \text { otherwise }\end{cases}
$$

with $1<p<\infty$, where we assume that $W: \Omega \times \mathbb{R}^{2} \times \mathbb{R}^{3 \times 3} \rightarrow \mathbb{R}$ satisfies the following hypotheses:

$\left(H_{1}\right) W(x, \cdot ; \cdot)$ is continuous for a.e. $x \in \Omega$;

$\left(H_{2}\right) W(\cdot, \cdot ; \xi)$ is $\mathcal{L}^{3} \otimes \mathcal{L}^{2}$-measurable for all $\xi \in \mathbb{R}^{3 \times 3}$;

$\left(H_{3}\right)$ there exists $0<\beta<+\infty$ such that

$$
\frac{1}{\beta}|\xi|^{p}-\beta \leqslant W\left(x, y_{\alpha} ; \xi\right) \leqslant \beta\left(1+|\xi|^{p}\right), \quad \text { for a.e. } x \in \Omega \text { and for all }\left(y_{\alpha}, \xi\right) \in \mathbb{R}^{2} \times \mathbb{R}^{3 \times 3}
$$

$\left(H_{4}\right) W(x, \cdot ; \xi)$ is $Q^{\prime}$-periodic for a.e. $x \in \Omega$ and all $\xi \in \mathbb{R}^{3 \times 3}$, where we denote by $Q^{\prime}=(0,1)^{2}$ the unit cube of $\mathbb{R}^{2}$.

Remark 1.1. We remark that due to hypothese $\left(H_{1}\right)$ and $\left(H_{2}\right)$ the function $W$ is a Carathéodory integrand as $W(x, \cdot ; \cdot)$ is continuous a.e. $x \in \Omega$ and $W\left(\cdot, y_{\alpha} ; \xi\right)$ is measurable for all $y_{\alpha} \in \mathbb{R}^{2}$ and $\xi \in \mathbb{R}^{3 \times 3}$. This implies (see e.g. Proposition 3.3 in Braides and Defranceschi [9] or Proposition 1.1, Chapter VIII in Ekeland and Temam [14]) that $W$ is equivalent to a Borel function, that is there exist a Borel function $\tilde{W}$ such that $W(x, \cdot ; \cdot)=\tilde{W}(x, \cdot ; \cdot)$ for a.e. $x \in \Omega$. As a consequence the integral in (1.2) is well defined. As noted by Allaire in [2], Section 5, the measurability of $W$ in the pair $\left(x, y_{\alpha}\right)$ does not let us conclude that, for fixed $\xi$, the function $x \mapsto W\left(x, x_{\alpha} / \varepsilon ; \xi\right)$ is measurable. The continuity of $W\left(x, y_{\alpha} ; \xi\right)$ in at least one of the variables $x$ or $y_{\alpha}$ turns out to be sufficient to guarantee the measurability of this function. In the present paper, we decide to impose the continuity in the $y_{\alpha}$ variable. Note that we could also have considered $W$ to be continuous in $x$ and measurable in $y_{\alpha}$ but the proof of our main result does not hold anymore in this context.

As for notation, we will identify $W^{1, p}\left(\omega ; \mathbb{R}^{3}\right)$ with the set of functions $u \in W^{1, p}\left(\Omega ; \mathbb{R}^{3}\right)$ such that $D_{3} u(x)=0$ for a.e. $x \in \Omega$ and we will use the notation $\Gamma\left(L^{p}(\Omega)\right.$ )-limit whenever we refer to the $\Gamma$-convergence with respect to the usual metric in $L^{p}\left(\Omega ; \mathbb{R}^{3}\right)$. We prove that

Theorem 1.2. If $W$ satisfies $\left(H_{1}\right),\left(H_{2}\right),\left(H_{3}\right)$ and $\left(H_{4}\right)$, then the family $\left\{\mathcal{I}_{\varepsilon}\right\}_{\varepsilon>0} \Gamma\left(L^{p}(\Omega)\right)$-converges to the functional $\mathcal{I}_{\text {hom }}: L^{p}\left(\Omega ; \mathbb{R}^{3}\right) \rightarrow \overline{\mathbb{R}}$ defined by

$$
\mathcal{I}_{\mathrm{hom}}(u):= \begin{cases}2 \int_{\omega} W_{\mathrm{hom}}\left(x_{\alpha} ; D_{\alpha} u\left(x_{\alpha}\right)\right) d x_{\alpha} & \text { if } u \in W^{1, p}\left(\omega ; \mathbb{R}^{3}\right), \\ +\infty & \text { otherwise }\end{cases}
$$

where $W_{\text {hom }}$ is given by 


$$
\begin{gathered}
W_{\text {hom }}\left(x_{\alpha} ; \bar{\xi}\right):=\lim _{T \rightarrow+\infty} \inf _{\varphi}\left\{\frac{1}{2 T^{2}} \int_{(0, T)^{2} \times I} W\left(x_{\alpha}, y_{3}, y_{\alpha} ; \bar{\xi}+D_{\alpha} \varphi(y) \mid D_{3} \varphi(y)\right) d y_{\alpha} d y_{3}:\right. \\
\left.\varphi \in W^{1, p}\left((0, T)^{2} \times I ; \mathbb{R}^{3}\right), \varphi=0 \text { on } \partial(0, T)^{2} \times I\right\}
\end{gathered}
$$

for a.e. $x_{\alpha} \in \omega$ and all $\bar{\xi} \in \mathbb{R}^{3 \times 2}$.

We note that the $p$-coercivity condition in $\left(H_{3}\right)$ ensures that the $\Gamma$-limit of $\mathcal{I}_{\varepsilon}$ remains unchanged if we consider the weak $W^{1, p}$-topology in place of the strong $L^{p}$-topology.

As a consequence of Theorem 1.2 we deduce the usual convergence of (quasi-)minimizers as stated in the following corollary.

Corollary 1.3. Let $\left\{u_{\varepsilon}\right\}$ be the sequence of quasi-minimizers defined in (1.1). Then $\left\{u_{\varepsilon}\right\}$ is weakly relatively compact in $W^{1, p}\left(\Omega ; \mathbb{R}^{3}\right)$. Furthermore, any limit point $u$ of this sequence is a solution of the minimization problem

$$
\min _{v-\left(x_{\alpha}, 0\right) \in W_{0}^{1, p}\left(\omega ; \mathbb{R}^{3}\right)}\left\{2 \int_{\omega} W_{\mathrm{hom}}\left(x_{\alpha} ; D_{\alpha} v\left(x_{\alpha}\right)\right) d x_{\alpha}-\int_{\omega}\left(\bar{f}+g^{+}+g^{-}\right)\left(x_{\alpha}\right) \cdot v\left(x_{\alpha}\right) d x_{\alpha}\right\},
$$

where $\bar{f}:=\frac{1}{2} \int_{I} f\left(\cdot, x_{3}\right) d x_{3}$ and $g^{ \pm}:=g(\cdot, \pm 1)$.

This departs from the classical result (see Proposition 7.2 in Braides and Defranceschi [9]) due to the presence of the boundary conditions. This difficulty is overcome thanks to Remark 3.2 which says that we can prescribe the lateral boundary condition of the recovery sequence. We do not include the proof of this corollary because it is similar to that of Corollary 1.3 in Bouchitté, Fonseca and Mascarenhas [8].

The plan of this work is as follows: In Section 2 we will discuss some properties of $W_{\text {hom }}$, namely that it is well defined, proving that the limit on the right hand side of (1.4) exists, and that $W_{\text {hom }}\left(x_{\alpha} ; \cdot\right)$ is continuous for a.e. $x_{\alpha} \in \omega$. Section 3 is devoted to the proof of our main result, Theorem 1.2. The starting point of our analysis is the $\Gamma$-limit integral representation result, Theorem 2.5 in Braides, Fonseca and Francfort [10]. Our objective is to identify the integrand, showing that is coincides (almost everywhere) with $W_{\text {hom }}$. An argument of two-scale convergence will be used to prove that the integrand of the $\Gamma$-limit is less than $W_{\text {hom }}$ in Lemma 3.4, taking an oscillating sequence of test functions for the $\Gamma$-limit (see also Baía and Fonseca [5]). On the other hand, since the problem at fixed $\varepsilon$ and the asymptotic problem are of different nature (a three-dimensional problem becoming a two-dimensional one), it appears a new difficulty to prove the converse inequality in Lemma 3.5. Indeed, arguing like in classical relaxation, we would use the Scorza-Dragoni Theorem (see assumptions $\left(H_{1}\right)$ and $\left.\left(H_{2}\right)\right)$ to find a compact set $K \subset \Omega$, the complement of which has arbitrarily small Lebesgue measure and such that $W$ is continuous on $K \times \mathbb{R}^{2} \times \mathbb{R}^{3 \times 3}$. To localize our functional on small cubes so as to apply a uniform continuity argument, we fix $x_{\alpha}^{0}$ in $\omega$ and for all $\left(x_{\alpha}, x_{3}\right) \in K$ we must ensure that $\left(x_{\alpha}^{0}, x_{3}\right) \in K$. But since $K$ is compact, we cannot expect this to be true, and this argument fails. To overcome this difficulty (see also Babadjian and Francfort [4]), we need to replace $W$ by a function which is (separately) continuous everywhere. That is the aim of Lemma 4.1 which provides a continuous extension of Carathéodory functions and will be proved in the Appendix in Section 4.

\section{Preliminary results}

In this section we will prove some properties of the stored energy $W_{\text {hom }}$ that will be of use in the proof of Theorem 1.2.

Remark 2.1. To prove Theorem 1.2 we may assume, without loss of generality, that $W$ is non negative. Indeed, in view of $\left(H_{3}\right)$ it suffices to replace $W$ by $W+\beta$. 
We begin by showing that in the definition (1.4) of $W_{\text {hom }}$ the limit as $T \rightarrow+\infty$ exists. The proof of this property is a direct consequence of a result due to Licht and Michaille [18], Theorem 3.1 (see also Lemma 4.3.6 in Bouchitté, Fonseca and Mascarenhas [7]). We introduce the following new condition :

$\left(H_{1}^{\prime}\right) W\left(x, y_{\alpha} ; \cdot\right)$ is continuous for a.e. $x \in \Omega$ and all $y_{\alpha} \in \mathbb{R}^{2}$.

Remark 2.2. Note that $\left(H_{1}\right)$ implies $\left(H_{1}^{\prime}\right)$. Furthermore, if $W$ satisfies $\left(H_{1}^{\prime}\right)$ and $\left(H_{2}\right), W$ is a Carathéodory function in the following sense : $W(\cdot, \cdot ; \xi)$ is $\mathcal{L}^{3} \otimes \mathcal{L}^{2}$-measurable for all $\xi \in \mathbb{R}^{3 \times 3}$ and $W\left(x, y_{\alpha} ; \cdot\right)$ is continuous for $\mathcal{L}^{3} \otimes \mathcal{L}^{2}$-a.e. $\left(x, y_{\alpha}\right) \in \Omega \times \mathbb{R}^{2}$. As a consequence, there exists a Borel function $W^{\prime}$ on $\Omega \times \mathbb{R}^{2} \times \mathbb{R}^{3 \times 3}$ such that $W\left(x, y_{\alpha} ; \cdot\right)=W^{\prime}\left(x, y_{\alpha} ; \cdot\right)$ for $\mathcal{L}^{3} \otimes \mathcal{L}^{2}$-a.e. $\left(x, y_{\alpha}\right) \in \Omega \times \mathbb{R}^{2}$. Thus the integral in (1.4) is well defined. We insist on the fact that, in principle, $W^{\prime}$ and $\tilde{W}$ (see Remark 1.1) need not to be equal.

Lemma 2.3. If $W$ satisfies $\left(H_{1}^{\prime}\right),\left(H_{2}\right),\left(H_{3}\right)$ and $\left(H_{4}\right)$, then

$$
\begin{aligned}
W_{\text {hom }}\left(x_{\alpha} ; \bar{\xi}\right)= & \lim _{T \rightarrow+\infty} \inf _{\varphi}\left\{\frac{1}{2 T^{2}} \int_{(0, T)^{2} \times I} W\left(x_{\alpha}, y_{3}, y_{\alpha} ; \bar{\xi}+D_{\alpha} \varphi(y) \mid D_{3} \varphi(y)\right) d y_{\alpha} d y_{3}:\right. \\
& \left.\varphi \in W^{1, p}\left((0, T)^{2} \times I ; \mathbb{R}^{3}\right), \varphi=0 \text { on } \partial(0, T)^{2} \times I\right\}
\end{aligned}
$$

exists for a.e. $x_{\alpha} \in \omega$ and all $\bar{\xi} \in \mathbb{R}^{3 \times 2}$.

Proof. Let $x_{\alpha} \in \omega$ be such that $\left(H_{1}^{\prime}\right),\left(H_{3}\right)$ and $\left(H_{4}\right)$ hold and let $\bar{\xi} \in \mathbb{R}^{3 \times 2}$. Define $\mu: \mathcal{A}\left(\mathbb{R}^{2}\right) \rightarrow \mathbb{R}^{+}$by

$$
\begin{gathered}
\mu(A):=\inf _{\varphi}\left\{\frac{1}{2} \int_{A \times I} W\left(x_{\alpha}, y_{3}, y_{\alpha} ; \bar{\xi}+D_{\alpha} \varphi(y) \mid D_{3} \varphi(y)\right) d y_{\alpha} d y_{3}:\right. \\
\left.\varphi \in W^{1, p}\left(A \times I ; \mathbb{R}^{3}\right), \varphi=0 \text { on } \partial A \times I\right\},
\end{gathered}
$$

where $\mathcal{A}\left(\mathbb{R}^{2}\right)$ stands for the family of open subsets of $\mathbb{R}^{2}$.

In view of Remark 2.2, $\mu$ is well defined and, thanks to $\left(H_{3}\right)$, it is a finite function. Moreover this set function satisfies the assumptions of Theorem $3.1 \mathrm{in} \mathrm{Licht} \mathrm{and} \mathrm{Michaille} \mathrm{[18].} \mathrm{Indeed} \mathrm{firstly,} \mathrm{by}\left(H_{3}\right), \mu(A) \leqslant \beta(1+$ $\left.|\bar{\xi}|^{p}\right) \mathcal{L}^{2}(A)$ for all $A \in \mathcal{A}\left(\mathbb{R}^{2}\right)$. Secondly, $\mu$ is subadditive, that is $\mu(C) \leqslant \mu(A)+\mu(B)$ for all $A, B, C \in \mathcal{A}\left(\mathbb{R}^{2}\right)$ with $A \cap B \neq \emptyset$ and $\bar{C}=\bar{A} \cup \bar{B}$. Finally, by $\left(H_{4}\right)$, for any $\mathbf{i} \in \mathbb{Z}^{2}, \mu(A+\mathbf{i})=\mu(A)$ for all $A \in \mathcal{A}\left(\mathbb{R}^{2}\right)$. As a consequence the limit

$$
\lim _{T \rightarrow+\infty} \frac{\mu\left((0, T)^{2}\right)}{T^{2}}=W_{\text {hom }}\left(x_{\alpha} ; \bar{\xi}\right)
$$

exists.

Remark 2.4. It can be proved that the limit as $T \rightarrow+\infty$ in (1.4) could be replaced by an infimum taken for every $T>0$ (see Braides and Defranceschi [9] or Baía and Fonseca [5]).

Now that $W_{\text {hom }}$ is well defined, we will show that $W_{\text {hom }}\left(x_{\alpha} ; \cdot\right)$ is continuous for a.e. $x_{\alpha} \in \omega$ for later use in Theorem 1.2. To prove this property directly it seems that we would need a little bit more than only the continuity condition imposed on $W\left(x, y_{\alpha} ; \cdot\right)$ (e.g. a $p$-Lipschitz condition). We remark that if $W\left(x, y_{\alpha} ; \cdot\right)$ was quasiconvex, then by the $p$-growth condition $\left(H_{3}\right), W\left(x, y_{\alpha} ; \cdot\right)$ would satisfy a $p$-Lipschitz condition (see Lemma 2.7 below). Since we do not want to a priori restrict too much the stored energy density, in order to compensate for this lack of regularity we prove first in Lemma 2.6 that the value of $W_{\text {hom }}$ does not change if we replace $W$ by its quasiconvexification $\mathcal{Q} W$ (see Remark 2.5 below). 
Remark 2.5. For a.e. $x \in \Omega$, all $y_{\alpha} \in \mathbb{R}^{2}$ and all $\xi \in \mathbb{R}^{3 \times 3}$ define

$$
\mathcal{Q} W\left(x, y_{\alpha} ; \xi\right):=\left[Q W\left(x, y_{\alpha} ; \cdot\right)\right](\xi)
$$

where $Q W\left(x, y_{\alpha} ; \cdot\right)$ stands for the usual quasiconvexification of $W\left(x, y_{\alpha} ; \cdot\right)$. Then, the function $\mathcal{Q} W\left(x, y_{\alpha} ; \cdot\right)$ is quasiconvex (see e.g. Dacorogna [12]) and if $W$ satisfies $\left(H_{1}\right),\left(H_{2}\right),\left(H_{3}\right)$ and $\left(H_{4}\right)$, so does $\mathcal{Q} W$ except that $\mathcal{Q} W(x, \cdot ; \xi)$ may only be upper semicontinuous (as the infimum of continuous functions) for a.e. $x \in \Omega$ and all $\xi \in \mathbb{R}^{3 \times 3}$. In particular, since $\mathcal{Q} W$ satisfies $\left(H_{1}^{\prime}\right),\left(H_{2}\right),\left(H_{3}\right)$ and $\left(H_{4}\right)$, by Lemma 2.3 it follows that

$$
\begin{gathered}
(\mathcal{Q} W)_{\text {hom }}\left(x_{\alpha} ; \bar{\xi}\right)=\lim _{T \rightarrow+\infty} \inf _{\varphi}\left\{\frac{1}{2 T^{2}} \int_{(0, T)^{2} \times I} \mathcal{Q} W\left(x_{\alpha}, y_{3}, y_{\alpha} ; \bar{\xi}+D_{\alpha} \varphi(y) \mid D_{3} \varphi(y)\right) d y_{\alpha} d y_{3}:\right. \\
\left.\varphi \in W^{1, p}\left((0, T)^{2} \times I ; \mathbb{R}^{3}\right), \varphi=0 \text { on } \partial(0, T)^{2} \times I\right\}
\end{gathered}
$$

exists for a.e. $x_{\alpha} \in \omega$ and all $\bar{\xi} \in \mathbb{R}^{3 \times 2}$.

Lemma 2.6. If $W$ satisfies $\left(H_{1}\right),\left(H_{2}\right),\left(H_{3}\right)$ and $\left(H_{4}\right)$, then $(\mathcal{Q} W)_{\text {hom }}\left(x_{\alpha} ; \bar{\xi}\right)=W_{\text {hom }}\left(x_{\alpha} ; \bar{\xi}\right)$ for a.e. $x_{\alpha} \in \omega$ and all $\bar{\xi} \in \mathbb{R}^{3 \times 2}$.

Proof. Let $x_{\alpha} \in \omega$ be such that both $(\mathcal{Q} W)_{\text {hom }}\left(x_{\alpha} ; \cdot\right)$ and $W_{\text {hom }}\left(x_{\alpha} ; \cdot\right)$ are well defined. Since $W \geqslant \mathcal{Q} W$, we have $W_{\text {hom }}\left(x_{\alpha} ; \bar{\xi}\right) \geqslant(\mathcal{Q} W)_{\text {hom }}\left(x_{\alpha} ; \bar{\xi}\right)$ for all $\bar{\xi} \in \mathbb{R}^{3 \times 2}$. Let us prove now the converse inequality. Let $\bar{\xi} \in \mathbb{R}^{3 \times 2}$. For each $n>0$, let $T_{n} \in \mathbb{N}$ and $\varphi_{n} \in W^{1, \infty}\left(\left(0, T_{n}\right)^{2} \times I ; \mathbb{R}^{3}\right)$ satisfying $\varphi_{n}=0$ on $\partial\left(0, T_{n}\right)^{2} \times I$, be such that

$$
(\mathcal{Q} W)_{\mathrm{hom}}\left(x_{\alpha} ; \bar{\xi}\right)+\frac{1}{n} \geqslant \frac{1}{2 T_{n}^{2}} \int_{\left(0, T_{n}\right)^{2} \times I} \mathcal{Q} W\left(x_{\alpha}, y_{3}, y_{\alpha} ; \bar{\xi}+D_{\alpha} \varphi_{n}(y) \mid D_{3} \varphi_{n}(y)\right) d y_{\alpha} d y_{3} .
$$

The Lipschitz regularity of $\varphi_{n}$ may be ensured because of the density of $W^{1, \infty}\left(\left(0, T_{n}\right)^{2} \times I ; \mathbb{R}^{3}\right)$ in $W^{1, p}\left(\left(0, T_{n}\right)^{2} \times\right.$ $I ; \mathbb{R}^{3}$ ) together with the $p$-growth condition $\left(H_{3}\right)$. Thus

$$
(\mathcal{Q} W)_{\text {hom }}\left(x_{\alpha} ; \bar{\xi}\right) \geqslant \limsup _{n \rightarrow+\infty} \frac{1}{2 T_{n}^{2}} \int_{\left(0, T_{n}\right)^{2} \times I} \mathcal{Q} W\left(x_{\alpha}, y_{3}, y_{\alpha} ; \bar{\xi}+D_{\alpha} \varphi_{n}(y) \mid D_{3} \varphi_{n}(y)\right) d y_{\alpha} d y_{3} .
$$

For each $n \in \mathbb{N}$ fixed, by Acerbi-Fusco Relaxation Theorem (see Lemma III.1 and Statement III.7 in [1]) and Remark 2.1, there exists a sequence $\left\{\varphi_{n, k}\right\}_{k} \subset W^{1, \infty}\left(\left(0, T_{n}\right)^{2} \times I ; \mathbb{R}^{3}\right)$ satisfying $\varphi_{n, k}=\varphi_{n}$ on $\partial\left[\left(0, T_{n}\right)^{2} \times I\right]$ with $\varphi_{n, k} \underset{k \rightarrow \infty}{\longrightarrow} \varphi_{n}$ and such that

$$
\begin{aligned}
& \frac{1}{2 T_{n}^{2}} \int_{\left(0, T_{n}\right)^{2} \times I} \mathcal{Q} W\left(x_{\alpha}, y_{3}, y_{\alpha} ; \bar{\xi}+D_{\alpha} \varphi_{n}(y) \mid D_{3} \varphi_{n}(y)\right) d y_{\alpha} d y_{3} \\
& =\lim _{k \rightarrow+\infty} \frac{1}{2 T_{n}^{2}} \int_{\left(0, T_{n}\right)^{2} \times I} W\left(x_{\alpha}, y_{3}, y_{\alpha} ; \bar{\xi}+D_{\alpha} \varphi_{n, k}(y) \mid D_{3} \varphi_{n, k}(y)\right) d y_{\alpha} d y_{3}
\end{aligned}
$$

From (2.1) we have

$$
\begin{aligned}
(\mathcal{Q} W)_{\text {hom }}\left(x_{\alpha} ; \bar{\xi}\right) & \geqslant \liminf _{n \rightarrow+\infty} \liminf _{k \rightarrow+\infty} \frac{1}{2 T_{n}^{2}} \int_{\left(0, T_{n}\right)^{2} \times I} W\left(x_{\alpha}, y_{3}, y_{\alpha} ; \bar{\xi}+D_{\alpha} \varphi_{n, k}(y) \mid D_{3} \varphi_{n, k}(y)\right) d y_{\alpha} d y_{3} \\
& \geqslant \liminf _{n \rightarrow \infty} \inf _{\varphi}\left\{\frac{1}{2 T_{n}^{2}} \int_{\left(0, T_{n}\right)^{2} \times I} W\left(x_{\alpha}, y_{3}, y_{\alpha} ; \bar{\xi}+D_{\alpha} \varphi(y) \mid D_{3} \varphi(y)\right) d y_{\alpha} d y_{3}:\right. \\
& \left.\quad \varphi \in W^{1, p}\left(\left(0, T_{n}\right)^{2} \times I ; \mathbb{R}^{3}\right), \varphi=0 \text { on } \partial\left(0, T_{n}\right)^{2} \times I\right\} \\
& =W_{\text {hom }}\left(x_{\alpha} ; \bar{\xi}\right) .
\end{aligned}
$$

We are now in position to prove the continuity of $W_{\text {hom }}$ in its second variable : 
Lemma 2.7. Let $W$ satisfying $\left(H_{1}\right),\left(H_{2}\right),\left(H_{3}\right)$ and $\left(H_{4}\right)$, then $W_{\mathrm{hom}}\left(x_{\alpha} ; \cdot\right)$ is continuous on $\mathbb{R}^{3 \times 2}$ for a.e. $x_{\alpha} \in \omega$.

Proof. We observe that by the $p$-growth condition in $\left(H_{3}\right)$ and Remark $2.5, \mathcal{Q} W$ satisfies a $p$-Lipschitz condition (see Marcellini [19]): There exists $\beta>0$ such that for all $y_{\alpha} \in \mathbb{R}^{2}$ and a.e. $x \in \Omega$,

$$
\left|\mathcal{Q} W\left(x, y_{\alpha} ; \xi_{1}\right)-\mathcal{Q} W\left(x, y_{\alpha} ; \xi_{2}\right)\right| \leqslant \beta\left(1+\left|\xi_{1}\right|^{p-1}+\left|\xi_{2}\right|^{p-1}\right)\left|\xi_{1}-\xi_{2}\right|, \quad \xi_{1}, \xi_{2} \in \mathbb{R}^{3 \times 3} .
$$

Take $x_{\alpha} \in \omega$ such that both $(\mathcal{Q} W)_{\text {hom }}\left(x_{\alpha} ; \cdot\right)$ and $W_{\text {hom }}\left(x_{\alpha} ; \cdot\right)$ are well defined. By Lemma 2.6 we have $(\mathcal{Q} W)_{\text {hom }}\left(x_{\alpha} ; \cdot\right)=W_{\text {hom }}\left(x_{\alpha} ; \cdot\right)$. Given $\bar{\xi} \in \mathbb{R}^{3 \times 2}$ let $\bar{\xi}_{n} \rightarrow \bar{\xi}$ in $\mathbb{R}^{3 \times 2}$. From the definition of $W_{\text {hom }}\left(x_{\alpha} ; \bar{\xi}\right)$, for fixed $\delta>0$ choose $T \in \mathbb{N}$ and $\varphi \in W^{1, p}\left((0, T)^{2} \times I ; \mathbb{R}^{3}\right), \varphi=0$ on $\partial(0, T)^{2} \times I$, such that

$$
W_{\mathrm{hom}}\left(x_{\alpha} ; \bar{\xi}\right)+\delta \geqslant \frac{1}{2 T^{2}} \int_{(0, T)^{2} \times I} W\left(x_{\alpha}, y_{3}, y_{\alpha} ; \bar{\xi}+D_{\alpha} \varphi(y) \mid D_{3} \varphi(y)\right) d y_{\alpha} d y_{3} .
$$

Therefore, Remark 2.4 yields

$$
\begin{aligned}
\limsup _{n \rightarrow+\infty} W_{\text {hom }}\left(x_{\alpha} ; \bar{\xi}_{n}\right) & \leqslant \limsup _{n \rightarrow+\infty} \frac{1}{2 T^{2}} \int_{(0, T)^{2} \times I} W\left(x_{\alpha}, y_{3}, y_{\alpha} ; \bar{\xi}_{n}+D_{\alpha} \varphi(y) \mid D_{3} \varphi(y)\right) d y_{\alpha} d y_{3} \\
& =\frac{1}{2 T^{2}} \int_{(0, T)^{2} \times I} W\left(x_{\alpha}, y_{3}, y_{\alpha} ; \bar{\xi}+D_{\alpha} \varphi(y) \mid D_{3} \varphi(y)\right) d y_{\alpha} d y_{3}
\end{aligned}
$$

due to hypothesis $\left(H_{1}\right)$, the $p$-growth condition in $\left(H_{3}\right)$ and Lebesgue's Dominated Convergence Theorem. So by (2.3) and letting $\delta \rightarrow 0$ we conclude that

$$
\limsup _{n \rightarrow+\infty} W_{\text {hom }}\left(x_{\alpha} ; \overline{\xi_{n}}\right) \leqslant W_{\text {hom }}\left(x_{\alpha} ; \bar{\xi}\right) .
$$

Similarly, for each $n \in \mathbb{N}$ consider $T_{n} \in \mathbb{N}\left(T_{n} \nearrow+\infty\right)$ and $\varphi_{n} \in W^{1, p}\left(\left(0, T_{n}\right)^{2} \times I ; \mathbb{R}^{3}\right), \varphi_{n}=0$ on $\partial\left(0, T_{n}\right)^{2} \times I$, such that

$$
\begin{aligned}
W_{\mathrm{hom}}\left(x_{\alpha} ; \bar{\xi}_{n}\right)+\frac{1}{n} & \geqslant \frac{1}{2 T_{n}^{2}} \int_{\left(0, T_{n}\right)^{2} \times I} \mathcal{Q} W\left(x_{\alpha}, y_{3}, y_{\alpha} ; \bar{\xi}_{n}+D_{\alpha} \varphi_{n}(y) \mid D_{3} \varphi_{n}(y)\right) d y_{\alpha} d y_{3} \\
& =\frac{1}{2} \int_{Q^{\prime} \times I} \mathcal{Q} W\left(x_{\alpha}, y_{3}, T_{n} y_{\alpha} ; \bar{\xi}_{n}+D_{\alpha} \varphi_{n}\left(T_{n} y_{\alpha}, y_{3}\right) \mid D_{3} \varphi_{n}\left(T_{n} y_{\alpha}, y_{3}\right)\right) d y_{\alpha} d y_{3} \\
& =\frac{1}{2} \int_{Q^{\prime} \times I} \mathcal{Q} W\left(x_{\alpha}, y_{3}, T_{n} y_{\alpha} ; \bar{\xi}_{n}+D_{\alpha} \psi_{n}(y) \mid T_{n} D_{3} \psi_{n}(y)\right) d y_{\alpha} d y_{3},
\end{aligned}
$$

after a change of variables and where $\psi_{n}(y):=\frac{1}{T_{n}} \varphi_{n}\left(T_{n} y_{\alpha}, y_{3}\right)$. Clearly the function $\psi_{n}$ belongs to $W^{1, p}\left(Q^{\prime} \times\right.$ $\left.I ; \mathbb{R}^{3}\right)$ and $\psi_{n}=0$ on $\partial Q^{\prime} \times I$. By the $p$-coercivity hypothesis in $\left(H_{3}\right)$ and $(2.4)$, the sequence $\left\{\left(D_{\alpha} \psi_{n} \mid T_{n} D_{3} \psi_{n}\right)\right\}$ is bounded in $L^{p}\left(Q^{\prime} \times I ; \mathbb{R}^{3 \times 3}\right)$ uniformly in $n$. We can write that

$$
\begin{aligned}
& \liminf _{n \rightarrow+\infty} \int_{Q^{\prime} \times I} \mathcal{Q} W\left(x_{\alpha}, y_{3}, T_{n} y_{\alpha} ; \bar{\xi}_{n}+D_{\alpha} \psi_{n}(y) \mid T_{n} D_{3} \psi_{n}(y)\right) d y_{\alpha} d y_{3} \\
& \geqslant \liminf _{n \rightarrow+\infty} \int_{Q^{\prime} \times I}\left[\mathcal{Q} W\left(x_{\alpha}, y_{3}, T_{n} y_{\alpha} ; \bar{\xi}_{n}+D_{\alpha} \psi_{n}(y) \mid T_{n} D_{3} \psi_{n}(y)\right)\right. \\
& \left.-\mathcal{Q} W\left(x_{\alpha}, y_{3}, T_{n} y_{\alpha} ; \bar{\xi}+D_{\alpha} \psi_{n}(y) \mid T_{n} D_{3} \psi_{n}(y)\right)\right] d y_{\alpha} d y_{3} \\
& \quad+\liminf _{n \rightarrow+\infty} \int_{Q^{\prime} \times I} \mathcal{Q} W\left(x_{\alpha}, y_{3}, T_{n} y_{\alpha} ; \bar{\xi}+D_{\alpha} \psi_{n}(y) \mid T_{n} D_{3} \psi_{n}(y)\right) d y_{\alpha} d y_{3} .
\end{aligned}
$$


Using (2.2), Hölder inequality, the fact that $\left\{\left\|\left(D_{\alpha} \psi_{n} \mid T_{n} D_{3} \psi_{n}\right)\right\|_{L^{p}\left(Q^{\prime} \times I ; \mathbb{R}^{3 \times 3}\right)}\right\}$ is bounded and $\bar{\xi}_{n} \rightarrow \bar{\xi}$, we obtain

$$
\begin{aligned}
\liminf _{n \rightarrow+\infty} \int_{Q^{\prime} \times I} & {\left[\mathcal{Q} W\left(x_{\alpha}, y_{3}, T_{n} y_{\alpha} ; \bar{\xi}_{n}+D_{\alpha} \psi_{n}(y) \mid T_{n} D_{3} \psi_{n}(y)\right)\right.} \\
& \left.-\mathcal{Q} W\left(x_{\alpha}, y_{3}, T_{n} y_{\alpha} ; \bar{\xi}+D_{\alpha} \psi_{n}(y) \mid T_{n} D_{3} \psi_{n}(y)\right)\right] d y_{\alpha} d y_{3}=0
\end{aligned}
$$

and consequently

$$
\begin{aligned}
\liminf _{n \rightarrow+\infty} W_{\text {hom }}\left(x_{\alpha} ; \bar{\xi}_{n}\right) & \geqslant \liminf _{n \rightarrow+\infty} \frac{1}{2} \int_{Q^{\prime} \times I} \mathcal{Q} W\left(x_{\alpha}, y_{3}, T_{n} y_{\alpha} ; \bar{\xi}+D_{\alpha} \psi_{n}(y) \mid T_{n} D_{3} \psi_{n}(y)\right) d y_{\alpha} d y_{3} \\
& =\liminf _{n \rightarrow+\infty} \frac{1}{2 T_{n}^{2}} \int_{\left(0, T_{n}\right)^{2} \times I} \mathcal{Q} W\left(x_{\alpha}, y_{3}, y_{\alpha} ; \bar{\xi}+D_{\alpha} \varphi_{n}(y) \mid D_{3} \varphi_{n}(y)\right) d y_{\alpha} d y_{3} \\
& \geqslant(\mathcal{Q} W)_{\text {hom }}\left(x_{\alpha} ; \bar{\xi}\right) \\
& =W_{\text {hom }}\left(x_{\alpha} ; \bar{\xi}\right) .
\end{aligned}
$$

From $(2.4)$ and $(2.5)$, we conclude that $W_{\text {hom }}\left(x_{\alpha} ; \cdot\right)$ is continuous at $\bar{\xi}$.

\section{Proof of Theorem 1.2}

We start by localizing our functionals. Representing by $\mathcal{A}(\omega)$ the class of all open subsets of $\omega$, define $\mathcal{I}_{\varepsilon}: L^{p}\left(\Omega ; \mathbb{R}^{3}\right) \times \mathcal{A}(\omega) \rightarrow \overline{\mathbb{R}}$ by

$$
\mathcal{I}_{\varepsilon}(u ; A):= \begin{cases}\int_{A \times I} W\left(x_{\alpha}, x_{3}, \frac{x_{\alpha}}{\varepsilon} ; D_{\alpha} u(x) \mid \frac{1}{\varepsilon} D_{3} u(x)\right) d x_{\alpha} d x_{3} & \text { if } u \in W^{1, p}\left(A \times I ; \mathbb{R}^{3}\right), \\ +\infty & \text { otherwise. }\end{cases}
$$

We will prove that the family of functionals $\left\{\mathcal{I}_{\varepsilon}(\cdot ; A)\right\}_{\varepsilon>0} \Gamma$-converges with respect to the $L^{p}\left(A \times I ; \mathbb{R}^{3}\right)$ topology to the functional $\mathcal{I}_{\text {hom }}(\cdot ; A): L^{p}\left(\Omega ; \mathbb{R}^{3}\right) \rightarrow \overline{\mathbb{R}}$

$$
\mathcal{I}_{\text {hom }}(u ; A):= \begin{cases}2 \int_{A} W_{\mathrm{hom}}\left(x_{\alpha} ; D_{\alpha} u\left(x_{\alpha}\right)\right) d x_{\alpha} & \text { if } u \in W^{1, p}\left(A ; \mathbb{R}^{3}\right), \\ +\infty & \text { otherwise }\end{cases}
$$

for all $A \in \mathcal{A}(\omega)$. As a consequence, taking $A=\omega$ yields Theorem 1.2.

For any $A \in \mathcal{A}(\omega)$ and any sequence $\left\{\varepsilon_{j}\right\} \searrow 0^{+}$, consider $\mathcal{I}_{\left\{\varepsilon_{j}\right\}}(\cdot ; A): L^{p}\left(\Omega ; \mathbb{R}^{3}\right) \rightarrow \overline{\mathbb{R}}$ the $\Gamma$-lower limit of $\left\{\mathcal{I}_{\varepsilon_{j}}(\cdot ; A)\right\}_{j \in \mathbb{N}}$

$$
\mathcal{I}_{\left\{\varepsilon_{j}\right\}}(u ; A):=\inf _{\left\{u_{j}\right\}}\left\{\liminf _{j \rightarrow+\infty} \mathcal{I}_{\varepsilon_{j}}\left(u_{j} ; A\right): u_{j} \rightarrow u \text { in } L^{p}\left(A \times I ; \mathbb{R}^{3}\right)\right\} .
$$

Remark 3.1. In view of the coercivity condition $\left(H_{4}\right)$, for all $A \in \mathcal{A}(\omega)$ we have that $\mathcal{I}_{\left\{\varepsilon_{j}\right\}}(u ; A)=+\infty$ whenever $u \in L^{p}\left(\Omega ; \mathbb{R}^{3}\right) \backslash W^{1, p}\left(A ; \mathbb{R}^{3}\right)$, hence our objective is to characterize $\mathcal{I}_{\left\{\varepsilon_{j}\right\}}(u ; A)$ for $u \in W^{1, p}\left(A ; \mathbb{R}^{3}\right)$.

By virtue of Remark 3.1, together with Theorem 2.5 in Braides, Fonseca and Francfort [10], it follows that every sequence $\left\{\varepsilon_{j}\right\}$ admits a subsequence $\left\{\varepsilon_{j_{n}}\right\} \equiv\left\{\varepsilon_{n}\right\}$ such that $\mathcal{I}_{\left\{\varepsilon_{n}\right\}}(\cdot ; A)$ defined in $(3.2)$ is the $\Gamma\left(L^{p}(A \times I)\right)$ limit of $\left\{\mathcal{I}_{\varepsilon_{n}}(\cdot ; A)\right\}_{n \in \mathbb{N}}$ for all $A \in \mathcal{A}(\omega)$. Further there exists a Carathéodory function $W_{\left\{\varepsilon_{n}\right\}}: \omega \times \mathbb{R}^{3 \times 2} \rightarrow \mathbb{R}$ such that 


$$
\mathcal{I}_{\left\{\varepsilon_{n}\right\}}(u ; A)=2 \int_{A} W_{\left\{\varepsilon_{n}\right\}}\left(x_{\alpha} ; D_{\alpha} u\left(x_{\alpha}\right)\right) d x_{\alpha},
$$

for all $A \in \mathcal{A}(\omega)$ and all $u \in W^{1, p}\left(A ; \mathbb{R}^{3}\right)$.

Our aim is to show that $\mathcal{I}_{\left\{\varepsilon_{n}\right\}}(\cdot ; A)=\mathcal{I}_{\text {hom }}(\cdot ; A)$ on $W^{1, p}\left(A ; \mathbb{R}^{3}\right)$ for all $A \in \mathcal{A}(\omega)$. Given $A \in \mathcal{A}(\omega)$, in view of the integral representation (3.3) and (3.1), it is enough to show that $W_{\left\{\varepsilon_{n}\right\}}\left(x_{\alpha} ; \bar{\xi}\right)=W_{\text {hom }}\left(x_{\alpha} ; \bar{\xi}\right)$ for a.e. $x_{\alpha} \in A$ and all $\bar{\xi} \in \mathbb{R}^{3 \times 2}$, and thus to work with affine functions instead of general Sobolev functions. We will prove that $W_{\left\{\varepsilon_{n}\right\}}\left(x_{\alpha} ; \bar{\xi}\right)=W_{\text {hom }}\left(x_{\alpha} ; \bar{\xi}\right)$ for a.e. $x_{\alpha} \in \omega$ and all $\bar{\xi} \in \mathbb{R}^{3 \times 2}$.

Remark 3.2. Lemma 2.6 of Braides, Fonseca and Francfort [10] implies that $\mathcal{I}_{\left\{\varepsilon_{j}\right\}}(u ; A)$ is unchanged if the approximating sequences $\left\{u_{j}\right\}$ are constrained to match the lateral boundary condition of their target, i.e. $u_{j} \equiv u$ on $\partial A \times I$.

From now onward, $\left\{\varepsilon_{n}\right\}$ will denote a subsequence of $\left\{\varepsilon_{j}\right\}$ for which the $\Gamma\left(L^{p}(A \times I)\right)$-limit of $\left\{\mathcal{I}_{\varepsilon_{n}}(\cdot ; A)\right\}_{n \in \mathbb{N}}$ exists and coincides with $\mathcal{I}_{\left\{\varepsilon_{n}\right\}}(\cdot ; A)$ for all $A \in \mathcal{A}(\omega)$.

For each $T>0$ consider $\mathcal{S}_{T}$ a countable set of functions in $\mathcal{C}^{\infty}\left([0, T]^{2} \times[-1,1] ; \mathbb{R}^{3}\right)$ that is dense in

$$
\mathcal{W}_{T}=\left\{\varphi \in W^{1, p}\left((0, T)^{2} \times I ; \mathbb{R}^{3}\right): \varphi=0 \text { on } \partial(0, T)^{2} \times I\right\} .
$$

Definition 3.3. Let $L$ be the set of Lebesgue points $x_{\alpha}^{0}$ for all functions

$$
\begin{gathered}
W_{\left\{\varepsilon_{n}\right\}}(\cdot ; \bar{\xi}), \quad W_{\mathrm{hom}}(\cdot ; \bar{\xi}) \\
x_{\alpha} \mapsto \int_{Q^{\prime} \times I} W\left(x_{\alpha}, y_{3}, T y_{\alpha} ; \bar{\xi}+D_{\alpha} \varphi\left(T y_{\alpha}, y_{3}\right) \mid D_{3} \varphi\left(T y_{\alpha}, y_{3}\right)\right) d y_{\alpha} d y_{3},
\end{gathered}
$$

with $T \in \mathbb{N}, \varphi \in \mathcal{S}_{T}$ and $\bar{\xi} \in \mathbb{Q}^{3 \times 2}$ where $W_{\text {hom }}\left(x_{\alpha}^{0} ; \cdot\right)$ is well defined.

We have that $\mathcal{L}^{2}(\omega \backslash L)=0$. Given $x_{\alpha}^{0} \in L$, we denote by $Q^{\prime}\left(x_{\alpha}^{0}, \delta\right)$ the cube in $\mathbb{R}^{2}$ centered in $x_{\alpha}^{0}$ and of side length $\delta>0$ where $\delta$ is small enough so that $Q^{\prime}\left(x_{\alpha}^{0}, \delta\right) \in \mathcal{A}(\omega)$.

To prove that $W_{\left\{\varepsilon_{n}\right\}}\left(x_{\alpha} ; \bar{\xi}\right)=W_{\text {hom }}\left(x_{\alpha} ; \bar{\xi}\right)$ for a.e. $x_{\alpha} \in \omega$ and all $\bar{\xi} \in \mathbb{R}^{3 \times 2}$ we first show in Lemmas 3.4 and 3.5 below that both functions coincide on $L \times \mathbb{Q}^{3 \times 2}$. The general case will only be treated at the end of that section using the Carathéodory property of both integrands.

Fix $\bar{\xi} \in \mathbb{Q}^{3 \times 2}$ and set $v(x):=\bar{\xi} \cdot x_{\alpha}$. By $(3.3)$ and $(3.4)$

$$
\begin{aligned}
W_{\left\{\varepsilon_{n}\right\}}\left(x_{\alpha}^{0} ; \bar{\xi}\right) & =\lim _{\delta \rightarrow 0} \frac{1}{\delta^{2}} \int_{Q^{\prime}\left(x_{\alpha}^{0}, \delta\right)} W_{\left\{\varepsilon_{n}\right\}}\left(x_{\alpha} ; \bar{\xi}\right) d x_{\alpha} \\
& =\lim _{\delta \rightarrow 0} \frac{\mathcal{I}_{\left\{\varepsilon_{n}\right\}}\left(v ; Q^{\prime}\left(x_{\alpha}^{0}, \delta\right)\right)}{2 \delta^{2}} .
\end{aligned}
$$

Lemma 3.4. $W_{\left\{\varepsilon_{n}\right\}}\left(x_{\alpha}^{0} ; \bar{\xi}\right) \leqslant W_{\text {hom }}\left(x_{\alpha}^{0} ; \bar{\xi}\right)$ for all $x_{\alpha}^{0} \in L$ and all $\bar{\xi} \in \mathbb{Q}^{3 \times 2}$.

Proof. Given $k \in \mathbb{N}$, let $T_{k} \in \mathbb{N}$ and $\varphi_{k} \in \mathcal{S}_{T_{k}}$ with $\varphi_{k}=0$ on $\partial\left(0, T_{k}\right)^{2} \times I$, be such that

$$
W_{\text {hom }}\left(x_{\alpha}^{0} ; \bar{\xi}\right)+\frac{1}{k} \geqslant \frac{1}{2 T_{k}^{2}} \int_{\left(0, T_{k}\right)^{2} \times I} W\left(x_{\alpha}^{0}, y_{3}, y_{\alpha} ; \bar{\xi}+D_{\alpha} \varphi_{k}(y) \mid D_{3} \varphi_{k}(y)\right) d y .
$$

This is possible because of the continuity properties $\left(H_{1}\right)$ of $W$, the growth conditions $\left(H_{3}\right)$ and the density of $\mathcal{S}_{T_{k}}$ in $\mathcal{W}_{T_{k}}$. Extend $\varphi_{k}$ periodically with period $T_{k}$ to $\mathbb{R}^{2} \times I$. For $x \in \mathbb{R}^{2} \times I$, define $u_{n}^{k}(x):=\bar{\xi} \cdot x_{\alpha}+\varepsilon_{n} \varphi_{k}\left(\frac{x_{\alpha}}{\varepsilon_{n}}, x_{3}\right)$. For fixed $k, u_{n}^{k} \rightarrow v$ in $L^{p}\left(Q^{\prime}\left(x_{\alpha}^{0}, \delta\right) \times I ; \mathbb{R}^{3}\right)$ as $n \rightarrow \infty$, hence, by (3.6) 


$$
\begin{gathered}
W_{\left\{\varepsilon_{n}\right\}}\left(x_{\alpha}^{0} ; \bar{\xi}\right) \leqslant \liminf _{\delta \rightarrow 0} \liminf _{n \rightarrow+\infty} \frac{1}{2 \delta^{2}} \int_{Q^{\prime}\left(x_{\alpha}^{0}, \delta\right) \times I} W\left(x_{\alpha}, x_{3}, \frac{x_{\alpha}}{\varepsilon_{n}} ; D_{\alpha} u_{n}^{k} \mid \frac{1}{\varepsilon_{n}} D_{3} u_{n}^{k}\right) d x_{\alpha} d x_{3} \\
=\liminf _{\delta \rightarrow 0} \liminf _{n \rightarrow+\infty} \frac{1}{2 \delta^{2}} \int_{Q^{\prime}\left(x_{\alpha}^{0}, \delta\right) \times I} W\left(x_{\alpha}, x_{3}, \frac{x_{\alpha}}{\varepsilon_{n}} ; \bar{\xi}+D_{\alpha} \varphi_{k}\left(\frac{x_{\alpha}}{\varepsilon_{n}}, x_{3}\right) \mid D_{3} \varphi_{k}\left(\frac{x_{\alpha}}{\varepsilon_{n}}, x_{3}\right)\right) d x_{\alpha} d x_{3} .
\end{gathered}
$$

Define

$$
h_{k}\left(x_{\alpha}, y_{\alpha}\right):=\int_{-1}^{1} W\left(x_{\alpha}, x_{3}, T_{k} y_{\alpha} ; \bar{\xi}+D_{\alpha} \varphi_{k}\left(T_{k} y_{\alpha}, x_{3}\right) \mid D_{3} \varphi_{k}\left(T_{k} y_{\alpha}, x_{3}\right)\right) d x_{3}, \quad \text { for } x_{\alpha} \in \omega \text { and } y_{\alpha} \in \mathbb{R}^{2} .
$$

The continuity of $W$ with respect to $y_{\alpha}$, its measurability and periodicity properties, and the fact that $T_{k} \in \mathbb{N}$ lead us to conclude that the function $h_{k} \in L^{1}\left(Q^{\prime}\left(x_{\alpha}^{0} ; \delta\right) ; \mathcal{C}_{\text {per }}\left(Q^{\prime}\right)\right)$ for fixed $\delta>0$, where $\mathcal{C}_{\text {per }}\left(Q^{\prime}\right)$ denotes the space of $Q^{\prime}$-periodic and continuous functions defined on $\mathbb{R}^{2}$ (see Lemma 5.3 in Allaire [2]). Lemma 5.2 in [2] together with Fubini's Theorem yields to

$$
\begin{aligned}
& \lim _{n \rightarrow+\infty} \int_{Q^{\prime}\left(x_{\alpha}^{0}, \delta\right) \times I} W\left(x_{\alpha}, x_{3}, \frac{x_{\alpha}}{\varepsilon_{n}} ; \bar{\xi}+D_{\alpha} \varphi_{k}\left(\frac{x_{\alpha}}{\varepsilon_{n}}, x_{3}\right) \mid D_{3} \varphi_{k}\left(\frac{x_{\alpha}}{\varepsilon_{n}}, x_{3}\right)\right) d x_{\alpha} d x_{3} \\
= & \lim _{n \rightarrow+\infty} \int_{Q^{\prime}\left(x_{\alpha}^{0}, \delta\right)} h_{k}\left(x_{\alpha}, \frac{x_{\alpha}}{T_{k} \varepsilon_{n}}\right) d x_{\alpha} \\
= & \int_{Q^{\prime}\left(x_{\alpha}^{0}, \delta\right)} \int_{Q^{\prime}} h_{k}\left(x_{\alpha}, y_{\alpha}\right) d y_{\alpha} d x_{\alpha} \\
= & \int_{Q^{\prime}\left(x_{\alpha}^{0}, \delta\right)} \int_{Q^{\prime} \times I} W\left(x_{\alpha}, x_{3}, T_{k} y_{\alpha} ; \bar{\xi}+D_{\alpha} \varphi_{k}\left(T_{k} y_{\alpha}, x_{3}\right) \mid D_{3} \varphi_{k}\left(T_{k} y_{\alpha}, x_{3}\right)\right) d y_{\alpha} d x_{3} d x_{\alpha} .
\end{aligned}
$$

Using (3.5) we have

$$
\begin{aligned}
& W_{\left\{\varepsilon_{n}\right\}}\left(x_{\alpha}^{0} ; \bar{\xi}\right) \\
& \leqslant \liminf _{\delta \rightarrow 0} \frac{1}{2 \delta^{2}} \int_{Q^{\prime}\left(x_{\alpha}^{0}, \delta\right)} \int_{Q^{\prime} \times I} W\left(x_{\alpha}, x_{3}, T_{k} y_{\alpha} ; \bar{\xi}+D_{\alpha} \varphi_{k}\left(T_{k} y_{\alpha}, x_{3}\right) \mid D_{3} \varphi_{k}\left(T_{k} y_{\alpha}, x_{3}\right)\right) d y_{\alpha} d x_{3} d x_{\alpha} \\
& =\frac{1}{2} \int_{Q^{\prime} \times I} W\left(x_{\alpha}^{0}, x_{3}, T_{k} y_{\alpha} ; \bar{\xi}+D_{\alpha} \varphi_{k}\left(T_{k} y_{\alpha}, x_{3}\right) \mid D_{3} \varphi_{k}\left(T_{k} y_{\alpha}, x_{3}\right)\right) d y_{\alpha} d x_{3} \\
& \leqslant W_{\text {hom }}\left(x_{\alpha}^{0} ; \bar{\xi}\right)+\frac{1}{k} .
\end{aligned}
$$

Letting $k \rightarrow \infty$ we assert the claim.

Note that the same proof could be used to prove Lemma 2.5 in Babadjian and Francfort [4].

Lemma 3.5. $W_{\left\{\varepsilon_{n}\right\}}\left(x_{\alpha}^{0} ; \bar{\xi}\right) \geqslant W_{\text {hom }}\left(x_{\alpha}^{0} ; \bar{\xi}\right)$ for all $x_{\alpha}^{0} \in L$ and all $\bar{\xi} \in \mathbb{Q}^{3 \times 2}$.

Proof. Let $\left\{v_{n}\right\} \subset W^{1, p}\left(Q^{\prime}\left(x_{\alpha}^{0}, \delta\right) \times I ; \mathbb{R}^{3}\right)$ be a recovering sequence of the $\Gamma$-limit, i.e.

$$
v_{n} \rightarrow 0 \text { in } L^{p}\left(Q^{\prime}\left(x_{\alpha}^{0}, \delta\right) \times I ; \mathbb{R}^{3}\right)
$$

and 


$$
\mathcal{I}_{\left\{\varepsilon_{n}\right\}}\left(v ; Q^{\prime}\left(x_{\alpha}^{0}, \delta\right)\right)=\lim _{n \rightarrow+\infty} \int_{Q^{\prime}\left(x_{\alpha}^{0}, \delta\right) \times I} W\left(x_{\alpha}, x_{3}, \frac{x_{\alpha}}{\varepsilon_{n}} ; \bar{\xi}+D_{\alpha} v_{n} \mid \frac{1}{\varepsilon_{n}} D_{3} v_{n}\right) d x_{\alpha} d x_{3} .
$$

According to Theorem 1.1 in Bocea and Fonseca [6], there exists a subsequence of $\left\{\varepsilon_{n}\right\}$ (not relabelled) and a sequence $\left\{u_{n}\right\} \subset W^{1, p}\left(Q^{\prime}\left(x_{\alpha}^{0}, \delta\right) \times I ; \mathbb{R}^{3}\right)$ such that, upon setting $E_{n}:=\left\{x \in Q^{\prime}\left(x_{\alpha}^{0}, \delta\right) \times I: u_{n}(x)=v_{n}(x)\right\}$, then

$$
\left\{\begin{array}{l}
u_{n} \rightarrow 0 \text { in } L^{p}\left(Q^{\prime}\left(x_{\alpha}^{0}, \delta\right) \times I ; \mathbb{R}^{3}\right), \\
\left\{\left|\left(D_{\alpha} u_{n} \mid \frac{1}{\varepsilon_{n}} D_{3} u_{n}\right)\right|^{p}\right\} \text { is equi-integrable, } \\
\lim _{n \rightarrow+\infty} \mathcal{L}^{3}\left(\left[Q^{\prime}\left(x_{\alpha}^{0}, \delta\right) \times I\right] \backslash E_{n}\right)=0 .
\end{array}\right.
$$

Thus, in view of the $p$-growth condition $\left(H_{3}\right)$ together with (3.7) and Remark 2.1,

$$
\begin{aligned}
\mathcal{I}_{\left\{\varepsilon_{n}\right\}}\left(v ; Q^{\prime}\left(x_{\alpha}^{0}, \delta\right)\right) \geqslant & \limsup _{n \rightarrow+\infty} \int_{E_{n}} W\left(x_{\alpha}, x_{3}, \frac{x_{\alpha}}{\varepsilon_{n}} ; \bar{\xi}+D_{\alpha} u_{n} \mid \frac{1}{\varepsilon_{n}} D_{3} u_{n}\right) d x_{\alpha} d x_{3} \\
= & \limsup _{n \rightarrow+\infty} \int_{Q^{\prime}\left(x_{\alpha}^{0}, \delta\right) \times I} W\left(x_{\alpha}, x_{3}, \frac{x_{\alpha}}{\varepsilon_{n}} ; \bar{\xi}+D_{\alpha} u_{n} \mid \frac{1}{\varepsilon_{n}} D_{3} u_{n}\right) d x_{\alpha} d x_{3} \\
& -\limsup _{n \rightarrow+\infty} \int_{\left[Q^{\prime}\left(x_{\alpha}^{0}, \delta\right) \times I\right] \backslash E_{n}} W\left(x_{\alpha}, x_{3}, \frac{x_{\alpha}}{\varepsilon_{n}} ; \bar{\xi}+D_{\alpha} u_{n} \mid \frac{1}{\varepsilon_{n}} D_{3} u_{n}\right) d x_{\alpha} d x_{3} \\
\geqslant & \limsup _{n \rightarrow+\infty} \int_{Q^{\prime}\left(x_{\alpha}^{0}, \delta\right) \times I} W\left(x_{\alpha}, x_{3}, \frac{x_{\alpha}}{\varepsilon_{n}} ; \bar{\xi}+D_{\alpha} u_{n} \mid \frac{1}{\varepsilon_{n}} D_{3} u_{n}\right) d x_{\alpha} d x_{3} .
\end{aligned}
$$

For any $h \in \mathbb{N}$, we split $Q^{\prime}\left(x_{\alpha}^{0}, \delta\right)$ into $h^{2}$ disjoints cubes $Q_{i, h}^{\prime}$ of side length $\delta / h$ so that $Q^{\prime}\left(x_{\alpha}^{0}, \delta\right)=\bigcup_{i=1}^{h^{2}} Q_{i, h}^{\prime}$ and

$$
\mathcal{I}_{\left\{\varepsilon_{n}\right\}}\left(v ; Q^{\prime}\left(x_{\alpha}^{0}, \delta\right)\right) \geqslant \limsup _{h \rightarrow+\infty} \limsup _{n \rightarrow+\infty} \sum_{i=1}^{h^{2}} \int_{Q_{i, h}^{\prime} \times I} W\left(x_{\alpha}, x_{3}, \frac{x_{\alpha}}{\varepsilon_{n}} ; \bar{\xi}+D_{\alpha} u_{n} \mid \frac{1}{\varepsilon_{n}} D_{3} u_{n}\right) d x .
$$

For every $\eta>0$ and $\lambda>0$, let $K_{\eta} \subset \Omega$ and $W^{\eta, \lambda}$ be given by Lemma 4.1 below (with $N=d=3, m=2$ and $f=W)$. Then

$$
\mathcal{L}^{3}\left(\Omega \backslash K_{\eta}\right)<\eta
$$

On the other hand, define

$$
R_{n}^{\lambda}:=\left\{x \in Q^{\prime}\left(x_{\alpha}^{0}, \delta\right) \times I:\left|\left(\bar{\xi}+D_{\alpha} u_{n}(x) \mid \frac{1}{\varepsilon_{n}} D_{3} u_{n}(x)\right)\right| \leqslant \lambda\right\} .
$$

Chebyshev's inequality implies that there exists a constant $C>0$ - which does not depend on $n$ or $\lambda$ - such that

$$
\mathcal{L}^{3}\left(\left[Q^{\prime}\left(x_{\alpha}^{0}, \delta\right) \times I\right] \backslash R_{n}^{\lambda}\right)<\frac{C}{\lambda^{p}} .
$$

Since $W$ and $W^{\eta, \lambda}$ coincide on $K_{\eta} \times \mathbb{R}^{2} \times \bar{B}(0, \lambda)$, where in the sequel the set $\bar{B}(0, \lambda)$ stands for the closed ball $\left\{\xi \in \mathbb{R}^{3 \times 3}:|\xi| \leqslant \lambda\right\}$ of $\mathbb{R}^{3 \times 3}$, we get in view of $(3.8)$ 


$$
\begin{gathered}
\mathcal{I}_{\left\{\varepsilon_{n}\right\}}\left(v ; Q^{\prime}\left(x_{\alpha}^{0}, \delta\right)\right) \geqslant \\
\limsup _{\lambda \rightarrow+\infty} \limsup _{\eta \rightarrow 0} \limsup _{h \rightarrow+\infty} \limsup _{n \rightarrow+\infty} \sum_{i=1}^{h^{2}} \int_{\left[Q_{i, h}^{\prime} \times I\right] \cap R_{n}^{\lambda} \cap K_{\eta}} W^{\eta, \lambda}\left(x_{\alpha}, x_{3}, \frac{x_{\alpha}}{\varepsilon_{n}} ; \bar{\xi}+D_{\alpha} u_{n} \mid \frac{1}{\varepsilon_{n}} D_{3} u_{n}\right) d x .
\end{gathered}
$$

By virtue of (4.1) below and (3.9),

$$
\sum_{i=1}^{h^{2}} \int_{\left(\left[Q_{i, h}^{\prime} \times I\right] \cap R_{n}^{\lambda}\right) \backslash K_{\eta}} W^{\eta, \lambda}\left(x_{\alpha}, x_{3}, \frac{x_{\alpha}}{\varepsilon_{n}} ; \bar{\xi}+D_{\alpha} u_{n} \mid \frac{1}{\varepsilon_{n}} D_{3} u_{n}\right) d x \leqslant \beta\left(1+\lambda^{p}\right) \eta \underset{\eta \rightarrow 0}{\longrightarrow} 0,
$$

uniformly in $(n, h)$, so that

$$
\begin{gathered}
\mathcal{I}_{\left\{\varepsilon_{n}\right\}}\left(v ; Q^{\prime}\left(x_{\alpha}^{0}, \delta\right)\right) \geqslant \\
\limsup _{\lambda \rightarrow+\infty} \limsup _{\eta \rightarrow 0} \limsup _{h \rightarrow+\infty} \limsup _{n \rightarrow+\infty} \sum_{i=1}^{h^{2}} \int_{\left[Q_{i, h}^{\prime} \times I\right] \cap R_{n}^{\lambda}} W^{\eta, \lambda}\left(x_{\alpha}, x_{3}, \frac{x_{\alpha}}{\varepsilon_{n}} ; \bar{\xi}+D_{\alpha} u_{n} \mid \frac{1}{\varepsilon_{n}} D_{3} u_{n}\right) d x .
\end{gathered}
$$

Fix $y_{\alpha} \in Q^{\prime}$. Since $W^{\eta, \lambda}\left(\cdot, y_{\alpha} ; \cdot\right)$ is continuous, it is uniformly continuous on $\bar{\Omega} \times \bar{B}(0, \lambda)$, and we define the modulus of continuity $\omega_{\eta, \lambda}: Q^{\prime} \times \mathbb{R}^{+} \rightarrow \mathbb{R}^{+}$by

$$
\omega_{\eta, \lambda}\left(y_{\alpha}, t\right):=\sup _{(x, \xi),\left(x^{\prime}, \xi^{\prime}\right) \in \bar{\Omega} \times \bar{B}(0, \lambda)}\left\{\left|W^{\eta, \lambda}\left(x, y_{\alpha} ; \xi\right)-W^{\eta, \lambda}\left(x^{\prime}, y_{\alpha} ; \xi^{\prime}\right)\right|:\left|(x ; \xi)-\left(x^{\prime} ; \xi^{\prime}\right)\right| \leqslant t\right\} .
$$

Then

$$
\left\{\begin{array}{l}
\omega_{\eta, \lambda}(\cdot, t) \text { is lower semicontinuous for all } t \in \mathbb{R}^{+}, \\
\omega_{\eta, \lambda}\left(y_{\alpha}, \cdot\right) \text { is continuous and increasing for all } y_{\alpha} \in Q^{\prime} \\
\omega_{\eta, \lambda}\left(y_{\alpha}, 0\right)=0 \text { for all } y_{\alpha} \in Q^{\prime}
\end{array}\right.
$$

and

$$
\left|W^{\eta, \lambda}\left(x, y_{\alpha} ; \xi\right)-W^{\eta, \lambda}\left(x^{\prime}, y_{\alpha} ; \xi^{\prime}\right)\right| \leqslant \omega_{\eta, \lambda}\left(y_{\alpha},\left|x-x^{\prime}\right|+\left|\xi-\xi^{\prime}\right|\right) \text { for all }(x, \xi),\left(x^{\prime}, \xi^{\prime}\right) \in \bar{\Omega} \times \bar{B}(0, \lambda) .
$$

The first property is a consequence of the fact that the supremum of continuous functions in lower semicontinuous, while the other ones are classical properties of moduli of continuity.

For all $t \in \mathbb{R}^{+}$, we extend $\omega_{\eta, \lambda}(\cdot, t)$ to $\mathbb{R}^{2}$ by $Q^{\prime}$-periodicity. Since $W^{\eta, \lambda}(x, \cdot ; \xi)$ is $Q^{\prime}$-periodic, inequality (3.11) holds for all $y_{\alpha} \in \mathbb{R}^{2}$. Consequently, for every $\left(x_{\alpha}, x_{3}\right) \in\left[Q_{i, h}^{\prime} \times I\right] \cap R_{n}^{\lambda}$ and every $x_{\alpha}^{\prime} \in Q_{i, h}^{\prime}$,

$$
\begin{aligned}
& \mid W^{\eta, \lambda}\left(x_{\alpha}, x_{3}, \frac{x_{\alpha}}{\varepsilon_{n}} ; \bar{\xi}+D_{\alpha} u_{n}\left(x_{\alpha}, x_{3}\right) \mid \frac{1}{\varepsilon_{n}} D_{3} u_{n}\left(x_{\alpha}, x_{3}\right)\right) \\
& -W^{\eta, \lambda}\left(x_{\alpha}^{\prime}, x_{3}, \frac{x_{\alpha}}{\varepsilon_{n}} ; \bar{\xi}+D_{\alpha} u_{n}\left(x_{\alpha}, x_{3}\right) \mid \frac{1}{\varepsilon_{n}} D_{3} u_{n}\left(x_{\alpha}, x_{3}\right)\right) \mid \\
& \leqslant \omega_{\eta, \lambda}\left(\frac{x_{\alpha}}{\varepsilon_{n}},\left|x_{\alpha}-x_{\alpha}^{\prime}\right|\right) \leqslant \omega_{\eta, \lambda}\left(\frac{x_{\alpha}}{\varepsilon_{n}}, \frac{\sqrt{2} \delta}{h}\right) .
\end{aligned}
$$


We get, after integration in $\left(x_{\alpha}, x_{3}, x_{\alpha}^{\prime}\right)$ and summation,

$$
\begin{aligned}
& \sum_{i=1}^{h^{2}} \frac{h^{2}}{\delta^{2}} \int_{Q_{i, h}^{\prime}}\left\{\int_{R_{n}^{\lambda} \cap\left[Q_{i, h}^{\prime} \times I\right]} \mid W^{\eta, \lambda}\left(x_{\alpha}, x_{3}, \frac{x_{\alpha}}{\varepsilon_{n}} ; \bar{\xi}+D_{\alpha} u_{n}\left(x_{\alpha}, x_{3}\right) \mid \frac{1}{\varepsilon_{n}} D_{3} u_{n}\left(x_{\alpha}, x_{3}\right)\right)\right. \\
& \left.-W^{\eta, \lambda}\left(x_{\alpha}^{\prime}, x_{3}, \frac{x_{\alpha}}{\varepsilon_{n}} ; \bar{\xi}+D_{\alpha} u_{n}\left(x_{\alpha}, x_{3}\right) \mid \frac{1}{\varepsilon_{n}} D_{3} u_{n}\left(x_{\alpha}, x_{3}\right)\right) \mid d x\right\} d x_{\alpha}^{\prime} \\
& \leqslant 2 \int_{Q^{\prime}\left(x_{\alpha}^{0}, \delta\right)} \omega_{\eta, \lambda}\left(\frac{x_{\alpha}}{\varepsilon_{n}}, \frac{\sqrt{2} \delta}{h}\right) d x_{\alpha} .
\end{aligned}
$$

Riemann-Lebesgue's Lemma applied to the $Q^{\prime}$-periodic function $\omega_{\eta, \lambda}(\cdot, \sqrt{2} \delta / h)$ yields,

$$
\lim _{n \rightarrow+\infty} 2 \int_{Q^{\prime}\left(x_{\alpha}^{0}, \delta\right)} \omega_{\eta, \lambda}\left(\frac{x_{\alpha}}{\varepsilon_{n}}, \frac{\sqrt{2} \delta}{h}\right) d x_{\alpha}=2 \delta^{2} \int_{Q^{\prime}} \omega_{\eta, \lambda}\left(x_{\alpha}, \frac{\sqrt{2} \delta}{h}\right) d x_{\alpha},
$$

and by Beppo-Levi's Monotone Convergence Theorem

$$
\lim _{h \rightarrow+\infty} 2 \delta^{2} \int_{Q^{\prime}} \omega_{\eta, \lambda}\left(x_{\alpha}, \frac{\sqrt{2} \delta}{h}\right) d x_{\alpha}=0 .
$$

Hence, denoting by $\limsup _{\lambda, \eta, h, n}$ the successive $\limsup _{\lambda \rightarrow+\infty} \limsup _{\eta \rightarrow 0} \limsup _{h \rightarrow+\infty} \limsup _{n \rightarrow+\infty}$

$$
\begin{gathered}
\mathcal{I}_{\left\{\varepsilon_{n}\right\}}\left(v ; Q^{\prime}\left(x_{\alpha}^{0}, \delta\right)\right) \geqslant \\
\limsup _{\lambda, \eta, h, n} \sum_{i=1}^{h^{2}} \frac{h^{2}}{\delta^{2}} \int_{Q_{i, h}^{\prime}}\left\{\int_{\left[Q_{i, h}^{\prime} \times I\right] \cap R_{n}^{\lambda}} W^{\eta, \lambda}\left(x_{\alpha}^{\prime}, x_{3}, \frac{x_{\alpha}}{\varepsilon_{n}} ; \bar{\xi}+D_{\alpha} u_{n}\left(x_{\alpha}, x_{3}\right) \mid \frac{1}{\varepsilon_{n}} D_{3} u_{n}\left(x_{\alpha}, x_{3}\right)\right) d x_{\alpha} d x_{3}\right\} d x_{\alpha}^{\prime} .
\end{gathered}
$$

Define the following sets which depend on all parameters $(\eta, \lambda, i, h, n)$ :

$$
\left\{\begin{array}{l}
T:=\left\{\left(x_{\alpha}^{\prime}, x_{\alpha}, x_{3}\right) \in Q_{i, h}^{\prime} \times Q_{i, h}^{\prime} \times I:\left(x_{\alpha}^{\prime}, x_{3}\right) \in K_{\eta} \text { and }\left(x_{\alpha}, x_{3}\right) \in R_{n}^{\lambda}\right\}, \\
T_{1}:=\left\{\left(x_{\alpha}^{\prime}, x_{\alpha}, x_{3}\right) \in Q_{i, h}^{\prime} \times Q_{i, h}^{\prime} \times I:\left(x_{\alpha}^{\prime}, x_{3}\right) \notin K_{\eta} \text { and }\left(x_{\alpha}, x_{3}\right) \in R_{n}^{\lambda}\right\}, \\
T_{2}:=\left\{\left(x_{\alpha}^{\prime}, x_{\alpha}, x_{3}\right) \in Q_{i, h}^{\prime} \times Q_{i, h}^{\prime} \times I:\left(x_{\alpha}, x_{3}\right) \notin R_{n}^{\lambda}\right\},
\end{array}\right.
$$

and note that $Q_{i, h}^{\prime} \times Q_{i, h}^{\prime} \times I=T \cup T_{1} \cup T_{2}$. Since $W\left(\cdot, y_{\alpha} ; \cdot\right)$ and $W^{\eta, \lambda}\left(\cdot, y_{\alpha} ; \cdot\right)$ coincide on $K_{\eta} \times \bar{B}(0, \lambda)$, we have

$$
\begin{aligned}
& \mathcal{I}_{\left\{\varepsilon_{n}\right\}}\left(v ; Q^{\prime}\left(x_{\alpha}^{0}, \delta\right)\right) \\
& \geqslant \limsup _{\lambda, \eta, h, n} \sum_{i=1}^{h^{2}} \frac{h^{2}}{\delta^{2}} \int_{T} W^{\eta, \lambda}\left(x_{\alpha}^{\prime}, x_{3}, \frac{x_{\alpha}}{\varepsilon_{n}} ; \bar{\xi}+D_{\alpha} u_{n}\left(x_{\alpha}, x_{3}\right) \mid \frac{1}{\varepsilon_{n}} D_{3} u_{n}\left(x_{\alpha}, x_{3}\right)\right) d x d x_{\alpha}^{\prime} \\
& \quad=\limsup _{\lambda, \eta, h, n} \sum_{i=1}^{h^{2}} \frac{h^{2}}{\delta^{2}} \int_{T} W\left(x_{\alpha}^{\prime}, x_{3}, \frac{x_{\alpha}}{\varepsilon_{n}} ; \bar{\xi}+D_{\alpha} u_{n}\left(x_{\alpha}, x_{3}\right) \mid \frac{1}{\varepsilon_{n}} D_{3} u_{n}\left(x_{\alpha}, x_{3}\right)\right) d x d x_{\alpha}^{\prime}
\end{aligned}
$$

We will prove that the corresponding terms over $T_{1}$ and $T_{2}$ are zero. Indeed, in view of (3.9) and the $p$-growth condition $\left(H_{3}\right)$, 


$$
\begin{aligned}
\sum_{i=1}^{h^{2}} \frac{h^{2}}{\delta^{2}} \int_{T_{1}} W\left(x_{\alpha}^{\prime},\right. & \left.x_{3}, \frac{x_{\alpha}}{\varepsilon_{n}} ; \bar{\xi}+D_{\alpha} u_{n}\left(x_{\alpha}, x_{3}\right) \mid \frac{1}{\varepsilon_{n}} D_{3} u_{n}\left(x_{\alpha}, x_{3}\right)\right) d x d x_{\alpha}^{\prime} \\
& \leqslant \sum_{i=1}^{h^{2}} \frac{h^{2}}{\delta^{2}} \mathcal{L}^{2}\left(Q_{i, h}^{\prime}\right) \mathcal{L}^{3}\left(\left[Q_{i, h}^{\prime} \times I\right] \backslash K_{\eta}\right) \beta\left(1+\lambda^{p}\right) \\
& <\beta\left(1+\lambda^{p}\right) \eta \underset{\eta \rightarrow 0}{\longrightarrow} 0
\end{aligned}
$$

uniformly in $(n, h)$. The bound from above in $\left(H_{3}\right)$, the equi-integrability of $\left\{\left|\left(D_{\alpha} u_{n} \mid \frac{1}{\varepsilon_{n}} D_{3} u_{n}\right)\right|^{p}\right\}$ and (3.10) imply that

$$
\begin{aligned}
\sum_{i=1}^{h^{2}} \frac{h^{2}}{\delta^{2}} \int_{T_{2}} W & \left(x_{\alpha}^{\prime}, x_{3}, \frac{x_{\alpha}}{\varepsilon_{n}} ; \bar{\xi}+D_{\alpha} u_{n}\left(x_{\alpha}, x_{3}\right) \mid \frac{1}{\varepsilon_{n}} D_{3} u_{n}\left(x_{\alpha}, x_{3}\right)\right) d x d x_{\alpha}^{\prime} \\
& \leqslant \sum_{i=1}^{h^{2}} \frac{h^{2}}{\delta^{2}} \mathcal{L}^{2}\left(Q_{i, h}^{\prime}\right) \beta \int_{\left[Q_{i, h}^{\prime} \times I\right] \backslash R_{n}^{\lambda}}\left(1+\left|\left(D_{\alpha} u_{n} \mid \frac{1}{\varepsilon_{n}} D_{3} u_{n}\right)\right|^{p}\right) d x \\
& =\beta \int_{\left[Q^{\prime}\left(x_{\alpha}^{0}, \delta\right) \times I\right] \backslash R_{n}^{\lambda}}\left(1+\left|\left(D_{\alpha} u_{n} \mid \frac{1}{\varepsilon_{n}} D_{3} u_{n}\right)\right|^{p}\right) d x \underset{\lambda \rightarrow+\infty}{\longrightarrow} 0,
\end{aligned}
$$

uniformly in $(\eta, n, h)$. Thus, in view of (3.12), (3.13), (3.14), Fatou's Lemma yields

$$
\begin{gathered}
\mathcal{I}_{\left\{\varepsilon_{n}\right\}}\left(v ; Q^{\prime}\left(x_{\alpha}^{0}, \delta\right)\right) \\
\geqslant \limsup _{h \rightarrow+\infty} \limsup _{n \rightarrow+\infty} \sum_{i=1}^{h^{2}} \frac{h^{2}}{\delta^{2}} \int_{Q_{i, h}^{\prime}} \int_{Q_{i, h}^{\prime} \times I} W\left(x_{\alpha}^{\prime}, x_{3}, \frac{x_{\alpha}}{\varepsilon_{n}} ; \bar{\xi}+D_{\alpha} u_{n}\left(x_{\alpha}, x_{3}\right) \mid \frac{1}{\varepsilon_{n}} D_{3} u_{n}\left(x_{\alpha}, x_{3}\right)\right) d x_{\alpha} d x_{3} d x_{\alpha}^{\prime} \\
\geqslant \limsup _{h \rightarrow+\infty} \sum_{i=1}^{h^{2}} \frac{h^{2}}{\delta^{2}} \int_{Q_{i, h}^{\prime}} \liminf _{n \rightarrow+\infty} \int_{Q_{i, h}^{\prime} \times I} W\left(x_{\alpha}^{\prime}, x_{3}, \frac{x_{\alpha}}{\varepsilon_{n}} ; \bar{\xi}+D_{\alpha} u_{n}\left(x_{\alpha}, x_{3}\right) \mid \frac{1}{\varepsilon_{n}} D_{3} u_{n}\left(x_{\alpha}, x_{3}\right)\right) d x_{\alpha} d x_{3} d x_{\alpha}^{\prime} .
\end{gathered}
$$

Fix $x_{\alpha}^{\prime} \in Q_{i, h}^{\prime}$ such that $W_{\text {hom }}\left(x_{\alpha}^{\prime} ; \bar{\xi}\right)$ is well defined and set $Z(x ; \xi):=W\left(x_{\alpha}^{\prime}, x_{3}, x_{\alpha} ; \xi\right)$. It is easy to check that $Z$ is a Carathéodory integrand hence, applying Theorem 4.2 of Braides, Fonseca and Francfort [10], we get since $u_{n} \rightarrow 0$ in $L^{p}\left(Q^{\prime}\left(x_{\alpha}^{0}, \delta\right) \times I ; \mathbb{R}^{3}\right)$,

$$
2 \frac{\delta^{2}}{h^{2}} \bar{Z}(\bar{\xi}) \leqslant \liminf _{n \rightarrow+\infty} \int_{Q^{\prime}\left(x_{\alpha}^{0}, \delta\right) \times I} Z\left(\frac{x_{\alpha}}{\varepsilon_{n}}, x_{3} ; \bar{\xi}+D_{\alpha} u_{n}(x) \mid \frac{1}{\varepsilon} D_{3} u_{n}(x)\right) d x
$$

where

$$
\begin{aligned}
\bar{Z}(\bar{\xi}):=\inf _{T>0, \varphi}\left\{\int_{(0, T)^{2} \times I} Z\left(x ; \bar{\xi}+D_{\alpha} \varphi(x) \mid D_{3} \varphi(x)\right) d x:\right. & \\
& \left.\varphi \in W^{1, p}\left((0, T)^{2} \times I ; \mathbb{R}^{3}\right), \quad \varphi=0 \text { on } \partial(0, T)^{2} \times I\right\} .
\end{aligned}
$$

In view of the previous formula together with (1.4) and Remark 2.4, we have that $\bar{Z}(\bar{\xi})=W_{\text {hom }}\left(x_{\alpha}^{\prime} ; \bar{\xi}\right)$. Then

$$
\liminf _{n \rightarrow+\infty} \int_{Q_{i, h}^{\prime} \times I} W\left(x_{\alpha}^{\prime}, x_{3}, \frac{x_{\alpha}}{\varepsilon_{n}} ; \bar{\xi}+D_{\alpha} u_{n}\left(x_{\alpha}, x_{3}\right) \mid \frac{1}{\varepsilon_{n}} D_{3} u_{n}\left(x_{\alpha}, x_{3}\right)\right) d x_{\alpha} d x_{3} \geqslant \frac{2 \delta^{2}}{h^{2}} W_{\mathrm{hom}}\left(x_{\alpha}^{\prime} ; \bar{\xi}\right),
$$


and so

$$
\mathcal{I}_{\left\{\varepsilon_{n}\right\}}\left(v ; Q^{\prime}\left(x_{\alpha}^{0}, \delta\right)\right) \geqslant \limsup _{h \rightarrow+\infty} \sum_{i=1}^{h^{2}} \frac{h^{2}}{\delta^{2}} \int_{Q_{i, h}^{\prime}} \frac{2 \delta^{2}}{h^{2}} W_{\mathrm{hom}}\left(x_{\alpha}^{\prime} ; \bar{\xi}\right) d x_{\alpha}^{\prime}=2 \int_{Q^{\prime}\left(x_{\alpha}^{0}, \delta\right)} W_{\mathrm{hom}}\left(x_{\alpha}^{\prime} ; \bar{\xi}\right) d x_{\alpha}^{\prime} .
$$

Dividing both sides of the previous inequality by $\delta^{2}$ and passing to the limit when $\delta \searrow 0^{+}$, we obtain by (3.4) and (3.6)

$$
W_{\left\{\varepsilon_{n}\right\}}\left(x_{\alpha}^{0} ; \bar{\xi}\right) \geqslant W_{\mathrm{hom}}\left(x_{\alpha}^{0} ; \bar{\xi}\right)
$$

Proposition 3.6. $W_{\left\{\varepsilon_{n}\right\}}\left(x_{\alpha} ; \bar{\xi}\right)=W_{\text {hom }}\left(x_{\alpha} ; \bar{\xi}\right)$ for a.e. $x_{\alpha} \in \omega$ and for all $\bar{\xi} \in \mathbb{R}^{3 \times 2}$.

Proof. Let $E$ be the intersection of the set $L$ (see Definition 3.3) with the subset of points $x_{\alpha}^{0} \in \omega$ where $W_{\left\{\varepsilon_{n}\right\}}\left(x_{\alpha}^{0} ; \cdot\right)$ and $W_{\text {hom }}\left(x_{\alpha}^{0} ; \cdot\right)$ are continuous (see Lemma 2.7). Then $\mathcal{L}^{2}(\omega \backslash E)=0$ and by Lemma 3.4 and 3.5 we have that $W_{\left\{\varepsilon_{n}\right\}}\left(x_{\alpha}^{0} ; \bar{\xi}\right)=W_{\text {hom }}\left(x_{\alpha}^{0} ; \bar{\xi}\right)$ for all $x_{\alpha}^{0} \in E$ and for all $\bar{\xi} \in \mathbb{Q}^{3 \times 2}$. Since $W_{\left\{\varepsilon_{n}\right\}}\left(x_{\alpha}^{0} ; \cdot\right)$ and $W_{\text {hom }}\left(x_{\alpha}^{0} ; \cdot\right)$ are continuous for each $x_{\alpha}^{0} \in E$, the equality $W_{\left\{\varepsilon_{n}\right\}}\left(x_{\alpha}^{0} ; \bar{\xi}\right)=W_{\text {hom }}\left(x_{\alpha}^{0} ; \bar{\xi}\right)$ holds true for all $x_{\alpha}^{0} \in E$ and all $\bar{\xi} \in \mathbb{R}^{3 \times 2}$.

Corollary 3.7. For any $A \in \mathcal{A}(\omega), \Gamma\left(L^{p}(A \times I)\right)-\lim _{\varepsilon \rightarrow 0} \mathcal{I}_{\varepsilon}(\cdot ; A)=\mathcal{I}_{\text {hom }}(\cdot ; A)$, where $\mathcal{I}_{\text {hom }}(\cdot ; A)$ is the functional defined in (3.1).

Proof. From Proposition 3.6 we can conclude that $\mathcal{I}_{\text {hom }}(\cdot ; A)$ is well defined and

$$
\Gamma\left(L^{p}(A \times I)\right)-\lim _{n \rightarrow+\infty} \mathcal{I}_{\varepsilon_{n}}(\cdot ; A)=\mathcal{I}_{\text {hom }}(\cdot ; A)
$$

for all $A \in \mathcal{A}(\omega)$ (see Remark 3.1). Since this limit does not depend upon the extracted subsequence, in view of Proposition 7.11 in Braides and Defranceschi [9], the whole sequence $\left\{\mathcal{I}_{\varepsilon}(\cdot ; A)\right\}_{\varepsilon>0} \Gamma\left(L^{p}(A \times I)\right)$-converges to $\mathcal{I}_{\text {hom }}(\cdot ; A)$ for each $A \in \mathcal{A}(\omega)$.

The proof of Theorem 1.2 comes as a consequence of Corollary 3.7 taking $A=\omega$.

\section{Appendix}

We now prove a technical result of extension of Carathéodory functions that was useful in the proof of Lemma 3.5. The argument used is very close to that of Theorem 1, Section 1.2 in Evans and Gariepy [15].

Lemma 4.1. Let $\Omega \subset \mathbb{R}^{N}$ be a bounded open set and $f: \Omega \times \mathbb{R}^{m} \times \mathbb{R}^{d \times N} \rightarrow \mathbb{R}$ a function such that

$$
\left\{\begin{array}{l}
f(x, \cdot ; \cdot) \text { is continuous for a.e. } x \in \Omega ; \\
f(\cdot, \cdot ; \xi) \text { is } \mathcal{L}^{N} \otimes \mathcal{L}^{m} \text {-measurable for all } \xi \in \mathbb{R}^{N} ; \\
f(x, \cdot ; \xi) \text { is }(0,1)^{m} \text {-periodic for a.e. } x \in \Omega \text { and all } \xi \in \mathbb{R}^{d \times N} .
\end{array}\right.
$$

Assume also that there exists $\beta>0$ and $0<p<\infty$ such that

$$
\frac{1}{\beta}|\xi|^{p}-\beta \leqslant f(x, y ; \xi) \leqslant \beta\left(1+|\xi|^{p}\right), \quad \text { for a.e. } x \in \Omega \text { and all }(y, \xi) \in \mathbb{R}^{m} \times \mathbb{R}^{d \times N} .
$$


Then for any $\eta>0$ and $\lambda>0$ there exist a compact set $K_{\eta} \subset \Omega$ and a function $f^{\eta, \lambda}: \mathbb{R}^{N} \times \mathbb{R}^{m} \times \mathbb{R}^{d \times N} \rightarrow \mathbb{R}$ such that

$$
\left\{\begin{array}{l}
\mathcal{L}^{N}\left(\Omega \backslash K_{\eta}\right)<\eta, \\
f^{\eta, \lambda}(x, y ; \xi)=f(x, y ; \xi) \text { for all }(x, y ; \xi) \in K_{\eta} \times \mathbb{R}^{m} \times \bar{B}(0, \lambda), \\
f^{\eta, \lambda}(\cdot, y ; \cdot) \text { is continuous for all } y \in \mathbb{R}^{m}, \\
f^{\eta, \lambda}(x, \cdot ; \xi) \text { is continuous and }(0,1)^{m} \text {-periodic for all }(x, \xi) \in \mathbb{R}^{N} \times \mathbb{R}^{d \times N},
\end{array}\right.
$$

and

$$
-\beta \leqslant f^{\eta, \lambda}(x, y ; \xi) \leqslant \beta\left(1+\lambda^{p}\right), \quad \text { for all }(x, y, \xi) \in \mathbb{R}^{N} \times \mathbb{R}^{m} \times \mathbb{R}^{d \times N} .
$$

Proof. Since $f$ is a Carathéodory function, by Scorza Dragoni's Theorem (see Ekeland and Teman [14]) for all $\eta>0$ there exists a compact set $K_{\eta} \subset \Omega$ satisfying $\mathcal{L}^{N}\left(\Omega \backslash K_{\eta}\right)<\eta$ and such that $f$ is continuous on $K_{\eta} \times \mathbb{R}^{m} \times \mathbb{R}^{d \times N}$. Let $C^{\eta, \lambda}:=K_{\eta} \times \bar{B}(0, \lambda) \equiv C$ (to simplify notation) and $U^{\eta, \lambda}:=\left(\mathbb{R}^{N} \times \mathbb{R}^{d \times N}\right) \backslash C^{\eta, \lambda} \equiv U$. Fix $(s, F) \in C$, and for all $(x, \xi) \in U$ set

$$
u_{(s, F)}^{\eta, \lambda}(x, \xi):=\max \left\{2-\frac{|(s, F)-(x, \xi)|}{\operatorname{dist}((x, \xi), C)}, 0\right\} \equiv u_{(s, F)}(x, \xi) .
$$

Clearly

$$
\left\{\begin{array}{l}
u_{(s, F)} \text { is continuous on } U \\
0 \leqslant u_{(s, F)} \leqslant 1 \\
u_{(s, F)}(x, \xi)=0 \text { if and only if }|(s, F)-(x, \xi)| \geqslant 2 \operatorname{dist}((x, \xi), C) .
\end{array}\right.
$$

Let $\left\{s_{j}^{\eta}\right\}_{j \geqslant 1} \equiv\left\{s_{j}\right\}_{j \geqslant 1}$ and $\left\{F_{j}^{\lambda}\right\}_{j \geqslant 1} \equiv\left\{F_{j}\right\}_{j \geqslant 1}$ be a countable dense family of subsets of $K_{\eta}$ and $\bar{B}(0, \lambda)$, respectively. Define

$$
\sigma^{\eta, \lambda}(x, \xi):=\sum_{j \geqslant 1} 2^{-j} u_{\left(s_{j}, F_{j}\right)}(x, \xi) \equiv \sigma(x, \xi) \quad \text { for all }(x, \xi) \in U .
$$

Since $\sigma$ is the uniform limit of a sequence of continuous functions in $U$, then $\sigma$ is continuous in $U$. Moreover, for all $(x, \xi) \in U, 0<\sigma(x, \xi) \leqslant 1$. Indeed, assume that $\sigma(x, \xi)=0$ for some $(x, \xi) \in U$. Then, for all $j \geqslant 1, u_{\left(s_{j}, F_{j}\right)}(x, \xi)=0$ and thus $\left|\left(s_{j}, F_{j}\right)-(x, \xi)\right| \geqslant 2 \operatorname{dist}((x, \xi), C)$. The density of $\left\{s_{j}, F_{j}\right\}$ in $C$ yields that $|(s, F)-(x, \xi)| \geqslant 2 \operatorname{dist}((x, \xi), C)$ for all $(s, F) \in C$. We obtain a contradiction if we choose $(s, F)$ to be those points of $C$ such that $\operatorname{dist}((x, \xi), C)=\operatorname{dist}((x, \xi),(s, F))$ so $\sigma(x, \xi)>0$ for all $(x, \xi) \in U$. Consequently, the function

$$
(x, \xi) \mapsto v_{k}(x, \xi) \equiv v_{k}^{\eta, \lambda}(x, \xi):=\frac{2^{-k} u_{\left(s_{k}, F_{k}\right)}(x, \xi)}{\sigma(x, \xi)}
$$

is well defined and continuous in $U$. Moreover it satisfies that

$$
0 \leqslant v_{k}(x, \xi) \leqslant 1, \quad \sum_{k \geqslant 1} v_{k}(x, \xi)=1 \quad \text { for all }(x, \xi) \in U .
$$

Fix $y \in \mathbb{R}^{m}$ and define the continuous extension of $f(\cdot, y ; \cdot)$ outside $C$ as 


$$
f^{\eta, \lambda}(x, y ; \xi)= \begin{cases}f(x, y, \xi) & \text { if }(x, \xi) \in C, \\ \sum_{k \geqslant 1} v_{k}(x ; \xi) f\left(s_{k}, y ; F_{k}\right) & \text { if }(x, \xi) \in U .\end{cases}
$$

Obviously, we have $f^{\eta, \lambda}(x, y ; \xi)=f(x, y ; \xi)$ for all $(x, y ; \xi) \in K_{\eta} \times \mathbb{R}^{m} \times \bar{B}(0, \lambda)$. On the other hand, if $(x, y, \xi)$ is such that $(x, \xi) \in U$, in view of the $p$-growth and the $p$-coercivity condition on $f$ we get that

$$
-\beta \leqslant f^{\eta, \lambda}(x, y ; \xi) \leqslant \sum_{k \geqslant 1} v_{k}(x ; \xi) \beta\left(1+\left|F_{k}\right|^{p}\right) \leqslant \beta\left(1+\lambda^{p}\right) .
$$

Since we have that

$$
\sup _{y \in \mathbb{R}^{m},(x, \xi) \in U}\left[\sum_{k \geqslant n}\left|2^{-k} u_{\left(s_{k}, F_{k}\right)}(x, \xi) f\left(s_{k}, y ; F_{k}\right)\right|\right] \leqslant \beta\left(1+\lambda^{p}\right) \sum_{k \geqslant n} 2^{-k} \underset{n \rightarrow+\infty}{\longrightarrow} 0,
$$

then the function

$$
(x, y ; \xi) \mapsto \sum_{k \geqslant 1} 2^{-k} u_{\left(s_{k}, F_{k}\right)}(x, \xi) f\left(s_{k}, y ; F_{k}\right)
$$

is continuous on $\left\{(x, y, \xi):(x, \xi) \in U, y \in \mathbb{R}^{m}\right\}$. In particular, for all $(x, \xi) \in \mathbb{R}^{N} \times \mathbb{R}^{d \times N}$ the function $f^{\eta, \lambda}(x, \cdot ; \xi)$ is continuous. Further, $f^{\eta, \lambda}(x, \cdot ; \xi)$ it is $(0,1)^{m}$-periodic because if $\mathbf{i} \in \mathbb{Z}^{m}$ then for $(x, \xi) \in U$

$$
f^{\eta, \lambda}(x, y+\mathbf{i} ; \xi)=\sum_{k \geqslant 1} v_{k}(x ; \xi) f\left(s_{k}, y+\mathbf{i} ; F_{k}\right)=\sum_{k \geqslant 1} v_{k}(x ; \xi) f\left(s_{k}, y ; F_{k}\right)=f^{\eta, \lambda}(x, y ; \xi) .
$$

Finally we prove the continuity of $f^{\eta, \lambda}\left(\cdot, y_{;} \cdot\right)$. By (4.2) it suffices to show that for all $(a, A) \in C$

$$
\lim _{U \ni(x, \xi) \rightarrow(a, A)} f^{\eta, \lambda}(x, y ; \xi)=f(a, y ; A) .
$$

As $\left\{\left(s_{j}, F_{j}\right)\right\}_{j \geqslant 1}$ is dense in $C$ and $f(\cdot, y ; \cdot)$ is continuous on $C$, for every $\varepsilon>0$ there exists $\delta>0$ such that $\left|f(a, y ; A)-f\left(s_{j}, y ; F_{j}\right)\right|<\varepsilon$ for all $j \geqslant 1$ with $\left|(a, A)-\left(s_{j}, F_{j}\right)\right|<\delta$. Assume that $|(x, \xi)-(a, A)|<\delta / 4$ and suppose that $j \geqslant 1$ is such that $\left|(a, A)-\left(s_{j}, F_{j}\right)\right| \geqslant \delta$. Then

$$
\delta \leqslant\left|(a, A)-\left(s_{j}, F_{j}\right)\right| \leqslant|(a, A)-(x, \xi)|+\left|(x, \xi)-\left(s_{j}, F_{j}\right)\right| \leqslant \frac{\delta}{4}+\left|(x, \xi)-\left(s_{j}, F_{j}\right)\right|,
$$

and thus

$$
\left|(x, \xi)-\left(s_{j}, F_{j}\right)\right| \geqslant \frac{3 \delta}{4}>2|(a, A)-(x, \xi)| \geqslant 2 \operatorname{dist}((x, \xi), C) .
$$

Consequently, $v_{j}(x, \xi)=0$ if $j$ is such that $\left|(a, A)-\left(s_{j}, F_{j}\right)\right| \geqslant \delta$, and so

$$
\left|f^{\eta, \lambda}(x, y ; \xi)-f(a, y ; A)\right| \leqslant \sum_{j \geqslant 1,\left|(a, A)-\left(s_{j}, F_{j}\right)\right|<\delta} v_{j}(x, \xi)\left|f\left(s_{j}, y ; F_{j}\right)-f(a, y ; A)\right|<\varepsilon,
$$

because non zero terms of the sum are those which satisfy $\left|f(a, y ; A)-f\left(s_{j}, y ; F_{j}\right)\right|<\varepsilon$. The continuity of $f^{\eta, \lambda}(\cdot, y ; \cdot)$ now follows.

Acknowledgements: The authors would like to thank Irene Fonseca and Gilles Francfort for suggesting them this research work, and for their fruitful comments and suggestions. We wish to thank the Center of Nonlinear Analysis for its hospitality and support. The research of M. Baía was partially supported by Fundação para a Ciência e Tecnologia (Grant PRAXIS XXI SFRH $\backslash$ BD $\backslash 1174 \backslash 2000$ ), Fundo Social Europeu, the Department of Mathematical Sciences of Carnegie Mellon University and its Center for Nonlinear Analysis. 


\section{References}

[1] E. Acerbi, N. Fusco : Semicontinuity problems in Calculus of Variations, Arch. Rat. Mech. Anal. 86 (1984) $125-145$.

[2] G. Allaire : Homogenization and two scale convergence, SIAM J. Math. Anal. 23 (1992) 1482-1518.

[3] G. Anzellotti, S. Baldo and D. Percivale : Dimension reduction in variational problems, asymptotic development in $\Gamma$-convergence and thin structures in elasticity, Asymptotic Anal. 9 (1994) 61-100.

[4] J.-F. Babadjian, G. A. Francfort : Spatial heterogeneity in 3D-2D dimensional reduction, to appear in ESAIM : Cont. Opt. Calc. Var.

[5] M. Baía, I. Fonseca : $\Gamma$-convergence of functionals with periodic integrands via 2 -scale convergence, to be submitted.

[6] M. Bocea, I. Fonseca : Equi-integrability results for 3D-2D dimension reduction problems, ESAIM : Cont. Opt. Calc. Var. 7 (2002) 443-470.

[7] G. Bouchitté, F. Fonseca, L. Mascarenhas : A global method for relaxation, Arch. Rat. Mech. Anal. 145 (1998) 51-98.

[8] G. Bouchitté, F. Fonseca, L. Mascarenhas : Bending moment in membrane theory, J. Elasticity 73 (2003) 75-99.

[9] A. Braides, A. Defranceschi : Homogenization of multiple integrals, Oxford lectures series in mathematics and its applications, Clarendon Press, Oxford (1998).

[10] A. Braides, I. Fonseca, G. Francfort : 3D-2D asymptotic analysis for inhomogeneous thin films, Indiana Univ. Math. J. 49 (2000) 1367-1404.

[11] A. Braides : $\Gamma$-convergence for beginners, Oxford Lecture Series in Mathematics and its Applications, 22, Oxford University Press, Oxford (2002).

[12] B. Dacorogna : Direct methods in the calculus of variations, Springer-Verlag (1989).

[13] G. Dal Maso : An introduction to $\Gamma$ - convergence, Progress in Nonlinear Differential Equations and their Applications, 8. Birkhäuser Boston, Inc., Boston, MA (1993).

[14] I. Ekeland, R. Temam : Analyse convexe et problèmes variationnels, Dunod, Gauthiers-Villars, Paris (1974).

[15] L.C. Evans, R.F. Gariepy : Measure theory and fine properties of functions, Boca Raton, CRC Press (1992).

[16] D. Fox, A. Raoult, J.C. Simo : A justification of nonlinear properly invariant plate theories, Arch. Rat. Mech. Anat. 25 (1992) 257-199.

[17] H. Le Dret, A. Raoult : The nonlinear membrane model as variational limit of nonlinear three-dimensional elasticity, J. Math. Pures, Appl. 74 (1995) 549-578.

[18] C. Licht, G. Michaille : Global-local subadditive ergodic theorems and application to homogenization in elasticity, Annales Math. Blaise Pascal 9 (2002) 21-62.

[19] P. Marcellini : Approximation of quasiconvex functions, and lower semicontinuity of multiple integrals, Manuscripta Math. 51 (1985) 1-28. 
[20] G. Nguetseng, A general convergence result for a functional related to the theory of homogenization, SIAM J. Math. Anal. 20 (1989) 608-623.

[21] G. Nguetseng, Asymptotic analysis for a stiff variational problem arising in mechanics, SIAM J. Math. Anal. 21 (1990) 1394-1414.

[22] Y. C. Shu : Heterogeneous thin film of martensitic materials, Arch. Rat. Mech. Anal. 153 (2000) 39-90.

Jean-François Babadjian

L.P.M.T.M., Université Paris Nord, 93430, Villetaneuse, France

E-mail address: jfb@galilee.univ-paris13.fr

Margarida Baía

Department of Mathematical Science, Carnegie Mellon University,

Pittsburgh, PA 15213, USA

E-mail address: mbaia@andrew.cmu.edu 